\title{
RCMFRDE: Refined Composite Multiscale Fluctuation-Based Reverse Dispersion Entropy for Feature Extraction of Ship-Radiated Noise
}

\author{
Yuxing Li $\mathbb{D}$, ${ }^{1,2}$ Shangbin Jiao $\mathbb{D}^{1,},{ }^{1,2}$ Bo Geng $\mathbb{D}^{1},{ }^{1}$ and Xinru Jiang $\mathbb{D}^{1}$ \\ ${ }^{1}$ School of Automation and Information Engineering, Xi'an University of Technology, Xi'an 710048, China \\ ${ }^{2}$ Shaanxi Key Laboratory of Complex System Control and Intelligent Information Processing, Xi'an University of Technology, \\ Xi'an 710048, China \\ Correspondence should be addressed to Shangbin Jiao; jiaoshangbin@xaut.edu.cn
}

Received 16 September 2021; Revised 11 November 2021; Accepted 2 December 2021; Published 16 December 2021

Academic Editor: Mohammad Yaghoub Abdollahzadeh Jamalabadi

Copyright (c) 2021 Yuxing Li et al. This is an open access article distributed under the Creative Commons Attribution License, which permits unrestricted use, distribution, and reproduction in any medium, provided the original work is properly cited.

Dispersion entropy (DE), as a newly proposed entropy, has achieved remarkable results in its application. In this paper, on the basis of DE, combined with coarse-grained processing, we introduce the fluctuation and distance information of signal and propose the refined composite multiscale fluctuation-based reverse dispersion entropy (RCMFRDE). As an emerging complexity analysis mode, RCMFRDE has been used for the first time for the feature extraction of ship-radiated noise signals to mitigate the loss caused by the misclassification of ships on the ocean. Meanwhile, a classification and recognition method combined with K-nearest neighbor (KNN) came into being, namely, RCMFRDE-KNN. The experimental results indicated that RCMFRDE has the highest recognition rate in the single feature case and up to $100 \%$ in the double feature case, far better than multiscale $\mathrm{DE}$ (MDE), multiscale fluctuation-based DE (MFDE), multiscale permutation entropy (MPE), and multiscale reverse dispersion entropy (MRDE), and all the experimental results show that the RCMFRDE proposed in this paper improves the separability of the commonly used entropy in the hydroacoustic domain.

\section{Introduction}

The ocean is one of the main battlefields of future high-tech warfare $[1,2]$. With the increasing development of electronic countermeasure technology, people can use signal processing technology to extract features of ship-radiated noise signals to complete military tasks such as target detection and target recognition [3], and these signal processing methods include wavelet transform (WT) [4], empirical mode decomposition (EMD) [5], and variational mode decomposition (VMD) [6]. However, the key step in the ship-radiated noise classification is the extracted features $[7,8]$. The stronger the separability of the extracted features, the better the classification effect of ship-radiated noise signals.

Most of the traditional feature extraction methods extract the characteristic parameters of the waveform structure, which can be directly extracted from the time domain, including the mean value, variance, and peak value [9]. The transformation from the time domain to the frequency domain also transforms complex waveforms in the time domain into relatively simple single frequency component distributions in the frequency domain $[10,11]$. Therefore, the low line spectrum features and broadband spectrum features in the power spectrum can also become effective features for ship-radiated noise target detection and recognition and have achieved good results in signal classification. In addition, there are also many other kinds of features, such as chaos feature [12], time-frequency joint feature $[13,14]$, correlation dimension $[15,16]$, and auditory feature [17].

Lempel-Ziv complexity (LZC) is a commonly used indicator to identify dynamics [18-20] and is increasingly used as a feature for signal classification in various fields and has found numerous applications in characterizing the randomness of biological and ship-radiated noise signals [21]. 
In a nutshell, the physical meaning of LZC is that it reflects the rate at which new patterns appear in a time series as the length increases. The higher the LZC value of a sequence, the more new changes occur in the sequence and the more disordered and uncertain the sequence is, while the lower the LZC value, the more regular the sequence is $[22,23]$. In addition, more and more improved algorithms based on LZC are constantly being proposed, such as DLZC [24] and PLZC [25].

Another commonly used feature is entropy, which was first proposed by Shannon in 1948 [26]. It has been widely concerned and applied since the beginning of the 21st century because of its excellent performance of quickly and effectively quantifying the complexity of time series. In 2002, Bandt and Pompe first proposed Permutation Entropy (PE) [27], which was quickly applied to characterize the randomness of information in various fields, and many scholars improved it on this basis, such as weighted PE (WPE) [28] and multiscale PE (MPE) [29]; compared with PE, these improved PE-based measures have more substantial stability. However, PE only considers the order of amplitude values, ignores some information of amplitude values, and does not mention the influence of medium amplitude of each embedded vector. In some time series with large differences, the same PE value will be obtained, which will affect the stability of the extracted features [30].

To overcome the deficiencies of PE, Rostaghi and Azami proposed dispersion entropy (DE) in 2016 [31]. As an entropy has been proposed in recent years, DE has been proved to be more effective in measuring signal complexity than PE and improved PE-based measures. DE not only considers the order of amplitude values but also quantizes them through different mapping methods. The medium amplitude in the vector will also affect the DE value according to its size, and small changes will not change its corresponding class label in the vector. Compared with PE and improved PEbased measures, DE also has more vital noise anti-interference ability [32-34]. Because of the excellent ability of DE as a feature to characterizing the randomness of signals, many DE-based improved algorithms have appeared in just a few years, such as fluctuation-based DE (FDE) [35], reverse $\mathrm{DE}$ ( $\mathrm{RDE}$ ) [36], and the combination of the two, namely, fluctuation-based reverse DE (FRDE) [7]. However, the study in [7] did not consider various modes at different scales.

On the basis of FRDE, we first introduced the concept of multiscale, where the same sequence can obtain multiple subsequences by multiscale operation, and then improved the multiscale calculation by introducing the concept of refined composite, which can divide each subsequence into multiple sequences and average the final obtained entropy value, and finally obtained a new entropy called RCMFRDE. Combined with the clustering methods for classification recognition, the common clustering methods are K-nearest neighbor (KNN) [37], K-mean clustering [38], etc. In this paper, we choose the K-nearest neighbor as the classifier. In addition, we can further improve the final calculated entropy by introducing fractional order $[34,37]$, but this may lead to a lower separability of the final result out instead due to the effect of the backward scattering entropy. Experimental results show that the classification method based on RCMFRDE has stronger separability compared with several other commonly used entropy-based classification methods.

The train of thought of the paper is as follows: Section 2 reveals formulas of RCMFRDE; Section 3 proposes a feature extraction method that comes from RCMFRDE; Sections 4 and 5 extract four types of ship-radiated noise signals under single feature and double features; ultimately, Section 6 embraces main conclusions obtained.

\section{RCMFRDE}

Step 1: for a known time series $A(j)=\left\{a_{j}(j=1,2, \ldots\right.$, $N)\}, x_{k}^{(s)}(k=1,2, \ldots, s)$ is the $k^{\text {th }}$ coarse-grained time series of $A(j)$ as follows:

$$
\begin{aligned}
x_{k}^{(s)} & =\frac{1}{s} \sum_{j=k+s(i-1)}^{i s+k-1} a_{j}, \quad(i=1,2, \ldots, h), \\
h & =\text { floor } \frac{N-(k-1)}{s},
\end{aligned}
$$

where $s$ is the scale factor (SF), and floor is the downward rounding function. For $x_{k}^{(s)}=\left\{x_{k, i}^{(s)}(i=1,2\right.$, $\ldots, h)\}, x_{k, i}^{(s)}$ is the $i^{\text {th }}$ element in the $k^{\text {th }}$ time series. Step 2: to map $X=\left\{x_{k}^{(s)}(k=1,2, \ldots, s)\right\}$ to $Y=\left\{y_{k}^{(s)}(k=1,2, \ldots, s)\right\}$, normal cumulative distribution function is employed, which can be described as

$$
y_{k}^{(s)}=\frac{1}{\sigma \sqrt{2 \pi}} \int_{-\infty}^{x_{k}^{(s)}} e^{-\left((t-\mu)^{2} / 2 \sigma^{2}\right)} \mathrm{d} t
$$

where $\mu$ and $\sigma^{2}$ represent expectation and variance of $x_{k}^{(s)}$, respectively, and the range of $y_{k}^{(s)}$ distributes from 0 to 1 .

Step 3: $y_{k, i}^{(s)}$ is mapped to $z_{k, i}^{(s)}$ by using linear transformation as follows:

$$
z_{k, i}^{(s)}=\operatorname{round}\left(c * y_{k, i}^{(s)}+0.5\right),
$$

where $c$ is the number of categories, round is the integer function, and $z_{k, i}^{(s)}$ is an integer range from 1 to $c$. And, $z_{k, i}^{(s)}(i=1,2, \ldots, h)$ can form time series $z_{k}^{(s)}$, which can be expressed as $z_{k}^{(s)}=\left\{z_{k, i}^{(s)}(i=1,2, \ldots, h)\right\}$.

Step 4: phase space reconstruction of $z_{k}^{(s)}$ according to embedding dimension $m$ and time delay $\tau$, and embedding vectors $z_{k, n}^{c}, z_{k, n+\tau}^{c}, \ldots, z_{k, n+(m-1) \tau}^{c}$ is created as follows:

$$
\begin{aligned}
& Z_{k}^{m, \tau}=\left\{z_{k, n}^{c}, z_{k, n+\tau}^{c}, \ldots, z_{k, n+(m-1) \tau}^{c}\right\}, \\
& (n=1,2, \ldots, h-(m-1) \tau) .
\end{aligned}
$$

Step 5: each embedding vector is mapped to fluctuation-based dispersion patterns $T_{k}^{m, \tau}$. After this, the total number of vectors is $V=h-(m-1) \tau$, with a maximum of $(2 c-1)^{(m-1)}$ types of possible fluctuationbased dispersion patterns. There are $m-1$ values in 
each vector, and each value ranges from $-c+1$ to $c-1$. Based on $m, c, \tau, T_{k}^{m, \tau}$ can be expressed as

$$
\begin{aligned}
& T_{k}^{m, \tau}=\left\{t_{k, n}^{c}, t_{k, n+\tau}^{c}, \ldots, t_{k, n+(m-1) \tau}^{c}\right\}, \\
&(n=1,2, \ldots, h-(m-1) \tau) .
\end{aligned}
$$

All the vectors contained in the $k^{\text {th }}$ matrix can be described as

$$
\left[\begin{array}{c}
\left\{t_{k, 1}^{c}, t_{k, 1+\tau}^{c}, \ldots, t_{k, 1+(m-1) \tau}^{c}\right\} \\
\vdots \\
\left\{t_{k, j}^{c}, t_{k, j+\tau}^{c}, \ldots, t_{k, j+(m-1) \tau}^{c}\right\} \\
\vdots \\
\left\{t_{k, V}^{c}, t_{k, V+\tau}^{c}, \ldots, t_{k, V+(m-1) \tau}^{c}\right\}
\end{array}\right] .
$$

Step 6: each $T_{k}^{m, \tau}$ corresponds to a fluctuation-based dispersion pattern $\pi_{v_{0}, v_{1}, \ldots, v_{m-2}}\left(t_{k, n}^{c}=v_{0}, t_{k, n+\tau}^{c}=v_{1}, \ldots\right.$, $\left.t_{k, n+(m-1) \tau}^{c}=v_{m-2}\right)$; then, the probability of each fluctuation-based dispersion pattern $P_{k}^{(s)}(k=1,2, \ldots, s)$ can be estimated by

$$
P_{k}^{(s)}=\frac{\operatorname{Num}\left\{i \mid i \leq h-(m-1) \tau, \pi_{v_{0}, v_{1}, \ldots, v_{m-2}}\right\}}{h-(m-1) \tau},
$$

where $\operatorname{Num}\left\{i \mid i \leq h-(m-1) \tau, \pi_{v_{0}, v_{1}, \ldots, v_{m-2}}\right\} \quad$ is the number of fluctuation-based dispersion pattern of $\pi_{v_{0}, v_{1}, \ldots, v_{m-2}}$ assigned to $Z_{k}^{m, \tau}$. Next, the final average dispersion pattern $\bar{P}\left(\pi_{v_{0}, v_{1}, \ldots, v_{m-2}}\right)$ can be computed as

$$
\bar{P}\left(\pi_{v_{0}, v_{1}, \ldots, v_{m-2}}\right)=\frac{1}{s} \sum_{k=1}^{s} P_{k}^{(s)}, \quad(k=1,2, \ldots, s) .
$$

Step 7: $\operatorname{RCMFRDE}(A, m, c, s)$ is expressed as

$$
\begin{aligned}
\operatorname{RCMFRDE}(A, m, c, s)= & \sum_{\pi=1}^{(2 c-1)^{(m-1)}}\left(\bar{P}\left(\pi_{v_{0}, v_{1}, \ldots, v_{m-2}}\right)\right. \\
& \left.-\frac{1}{(2 c-1)^{(m-1)}}\right)^{2} .
\end{aligned}
$$

Step 8: the normalized RCMFRDE is defined as

$$
\operatorname{RCMFRDE}=\frac{\operatorname{RCMFRDE}(A, m, c, s)}{1-\left(1 /(2 c-1)^{(m-1)}\right)} .
$$

\section{Feature Extraction Method}

In this section, single feature extraction and double feature extraction are carried out, and Figure 1 shows the flowcharts of extracting features.

Detailed steps of extracting a single feature based on the method of RCMFRDE are as follows:

Step 1: four different types of S-NS are gathered for the comparison experiments of feature extraction.
Step 2: for each type of S-NS, 350 samples were randomly selected from them, and single feature extraction (from SF1 to SF5) was performed using RCMFRDE. To compare the various feature extraction methods, we performed the same operation for MDE, MFDE, MPE, and MRDE.

Step 3: KNN is used for classification. For each type of S-NS, the first 50 samples are considered as training samples, and the remaining 300 samples are considered as test samples.

Step 4: finally, the recognition rates of RCMFRDE (from SF1 to SF5) were obtained and compared with the other four feature extraction methods.

As shown in Figure 1(b), detailed steps of the double feature extraction method based on RCMFRDE are different from that of the single feature, especially in the number of features. There are four cases in which SF1 is combined with other scales for double feature extraction, including SF1 and SF2, SF1 and SF3, SF1 and SF4, and SF1 and SF5.

\section{Single Feature Extraction of the Four Types of S-NS}

4.1. Ship-Radiated Noise Signals. In this section, four types of S-NS are taken as the research object, called Ship I, Ship II, Ship III, and Ship IV, respectively. Each of the above S-NS lengths is $1.11 \times 10^{6}$, and the frequency of sample extraction is $44.1 \mathrm{kHz}$. The normalized results of each type of S-NS are represented in Figure 2.

4.2. Single Feature Extraction. In the experiment of single feature extraction, for each type of S-NS, 350 samples are selected, and each sample consists of 3,000 sampling points. Five kinds of entropies are employed to extract the complexity feature of each type of S-NS (from SF1 to SF5), including RCMFRDE, MDE, MFDE, MPE, and MRDE. Table 1 is the parameter settings of five kinds of entropies. For RCMFRDE, MDE, MFDE, MPE, and MRDE, time delay $\tau$ and embedding dimension $\mathrm{m}$ are 1 and 4, respectively. Except that MPE has no category number and mapping method, the category number and mapping method of other entropies are the same. Figures 3 to 7 demonstrate the distribution of five kinds of entropies for four types of S-NS for different scale factors.

From Figures 3 to 7 , it can be demonstrated that the different entropy distributions of the four types of S-NS for different scale factors are at different levels; for MDE and MFDE, with the increase of scale, the coincidence of MDE and MFDE distributions of Ship I, Ship II, and Ship III becomes more and more obvious, especially for Ship I and Ship II; when the SF is 4 and 5, the MPE distributions of S-NS with 4 different scale factors are more independent and have less overlap; for RCMFRDE and MRDE, when the SF is 1 , the distribution of S-NS for each type is at different levels for each scale factor, and the distribution of features for four types of S-NS is obviously different.

As evidenced by Table 2, the number of misidentified samples and the average recognition rate of the four S-NSs 


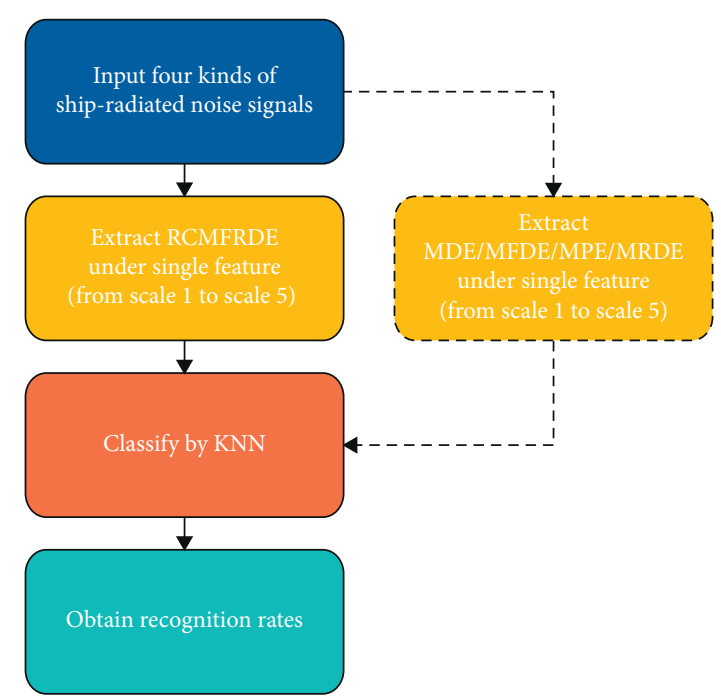

(a)

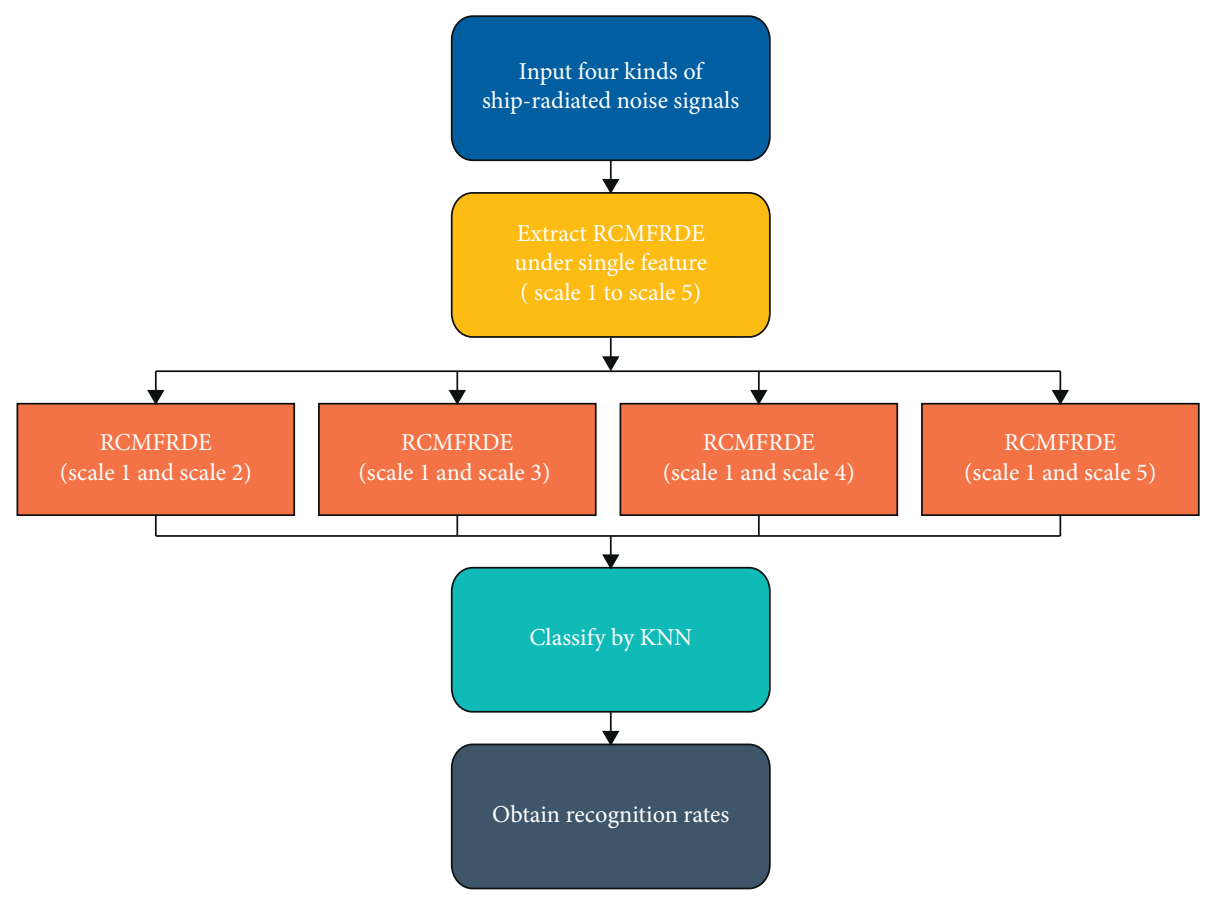

(b)

FIgURE 1: The flowcharts of extracting features. (a) Single feature. (b) Double feature.

under different entropies and different scale factors differ to different degrees; for RCMFRDE, three samples of ship IV are misidentified when the scale is 1 ; with the increase of scale, the number of misidentified samples of ship I, ship II, and ship III gradually increases, leading to a decrease in the average recognition rate of RCMFRDE; for MDE and MFDE, the three samples of ship IV are incorrectly recognized when SF is 1, while ship IV can be correctly recognized under other scale factors. We can conclude that the same feature extraction method has different recognition abilities for various types of S-NS under different scale factor conditions.

The experimental results proved that for all entropies, RCMFRDE has the highest recognition rate at scale 1, up to
$99.75 \%$, which is $10.19 \%$ higher than the other average recognition rates. However, while using only one feature, four types of S-NS cannot be fully recognized by RCMFRDE. For the sake of reducing the number of error samples and further improving the average recognition rate, the experiment of double feature extraction is carried out.

\section{Double Feature Extraction of the Four Types of S-NS}

5.1. Double Feature Extraction. The feature (SF1) with the highest average recognition rate of RCMFRDE is combined with other scale factors for double feature extraction. There are four cases, including SF1 and SF2, SF1 and SF3, SF1 and 


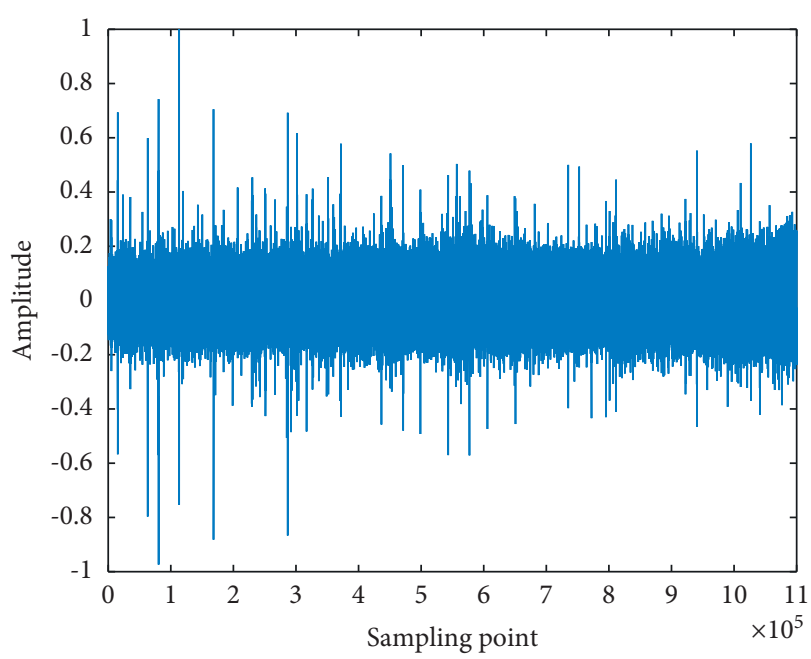

(a)

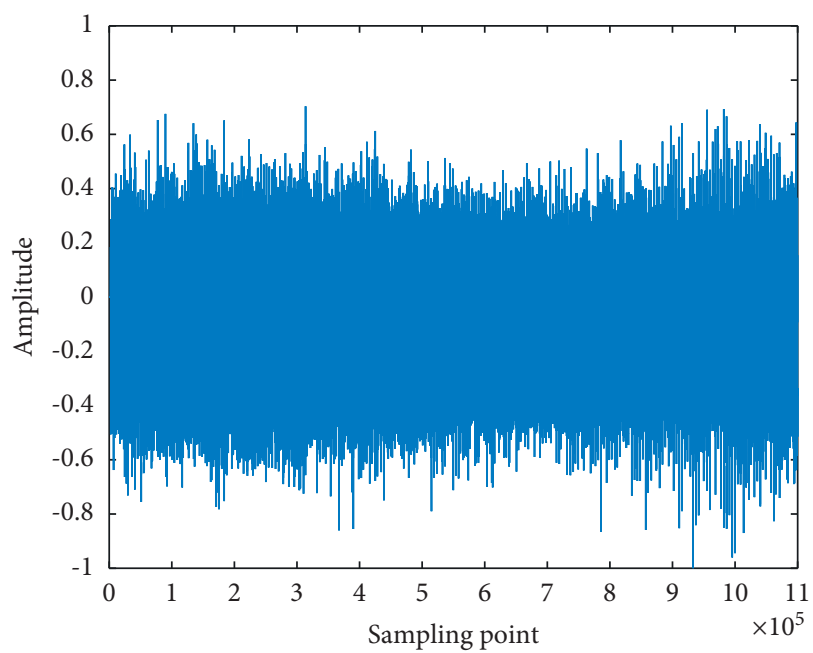

(c)

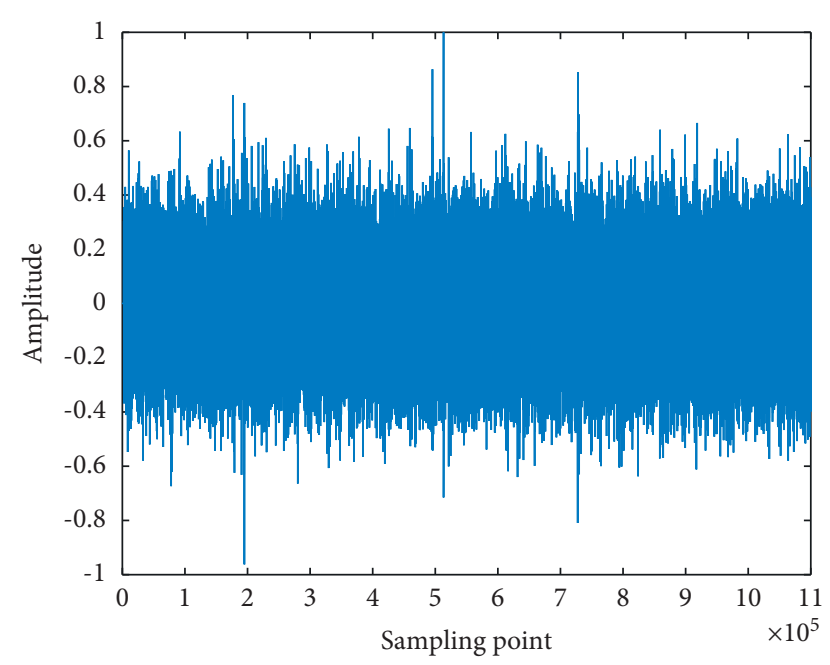

(b)

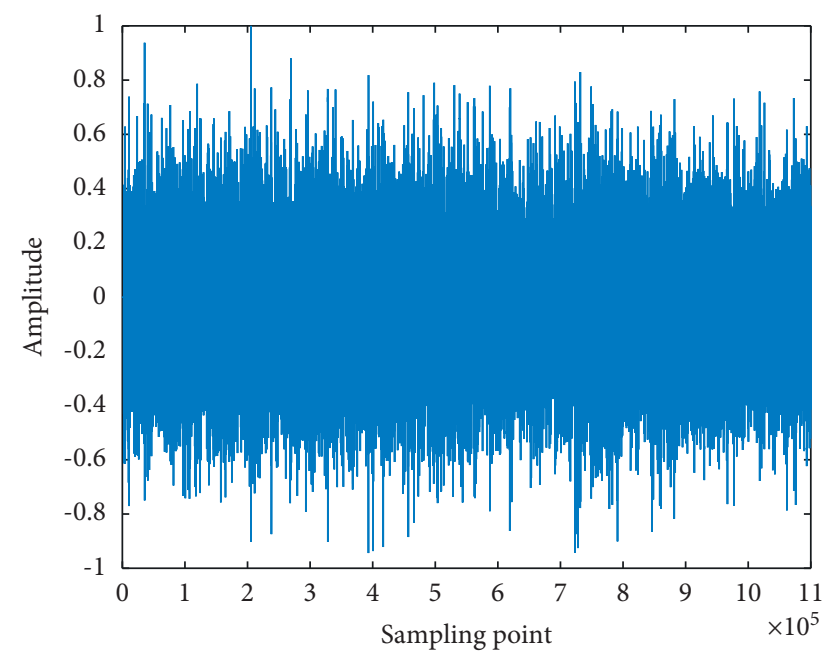

(d)

FIgure 2: The normalized results of four types of S-NS. (a) Ship I. (b) Ship II. (c) Ship III. (d) Ship IV.

TABLE 1: Parameter settings of five kinds of entropies.

\begin{tabular}{|c|c|c|c|c|c|}
\hline Entropy & Embedding dimension $m$ & Time delay $\tau$ & Category number $c$ & Mapping method & Scale s \\
\hline RCMFRDE & 4 & 1 & 5 & NCDF & $1,2,3,4,5$ \\
\hline $\mathrm{MDE}$ & 4 & 1 & 5 & $\mathrm{NCDF}$ & $1,2,3,4,5$ \\
\hline MFDE & 4 & 1 & 5 & NCDF & $1,2,3,4,5$ \\
\hline MPE & 4 & 1 & - & - & $1,2,3,4,5$ \\
\hline MRDE & 4 & 1 & 5 & NCDF & $1,2,3,4,5$ \\
\hline
\end{tabular}

SF4, and SF1 and SF5. Figures 8 to 12, respectively, express the distribution of five kinds of entropies for four types of S-NS under double features.

According to Figures 8 to 12, for the five kinds of entropies, Ship I and Ship IV are obviously different and easy to distinguish, while the difference between Ship II and Ship III is not obvious and difficult to distinguish; for Ship II and Ship III, RCMFRDE, MDE, and MFDE can distinguish them easily in all four cases, while other entropies cannot distinguish them completely.
5.2. Double Feature Classification and Recognition. For further comparing the double feature extraction effects of RCMFRDE, MDE, MFDE, MPE, and MRDE, KNN is applied to classify four types of S-NS, similar to feature classification and recognition, in which $\mathrm{K}$ value is selected as 1. Table 3 is the classification and recognition results of four types of S-NS under double features.

As shown in Table 3, only RCMFRDE can fully identify Ship III; all entropies except MRDE can fully identify Ship IV; among all combinations of entropies, the RCMFRDE- 

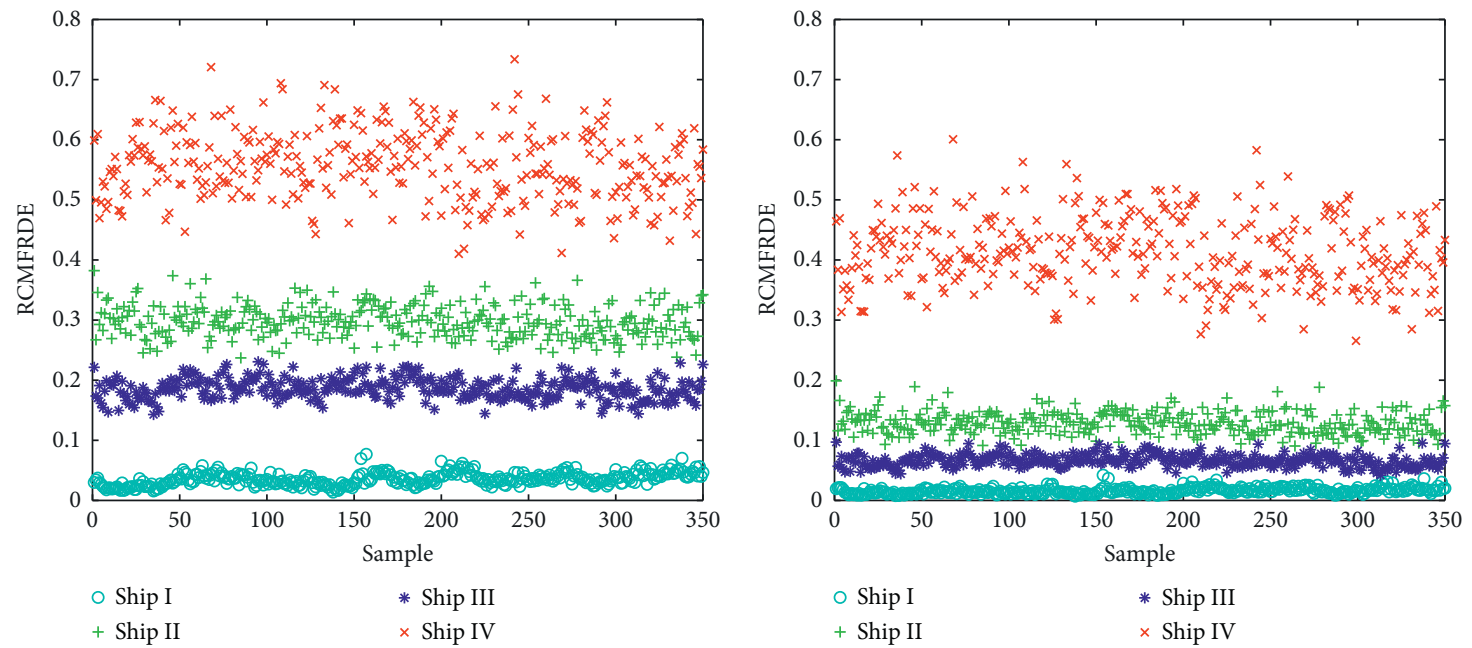

(a)

(b)

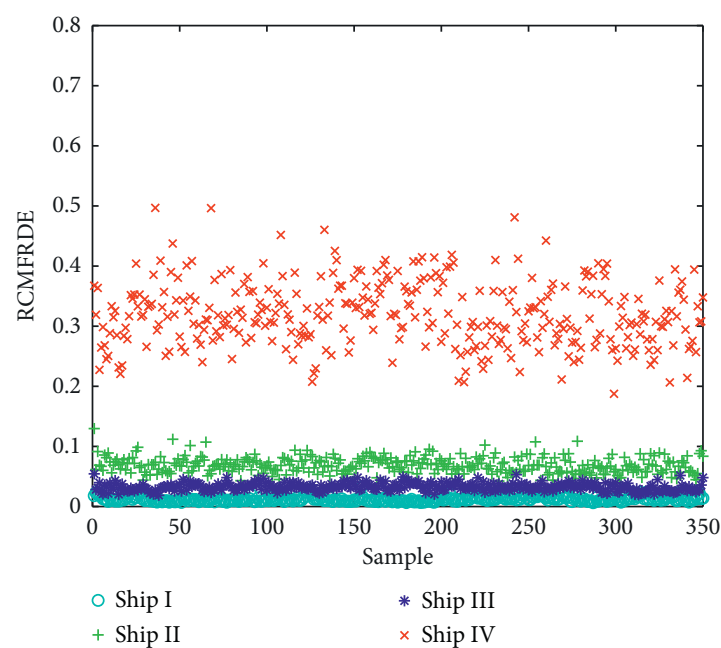

(c)

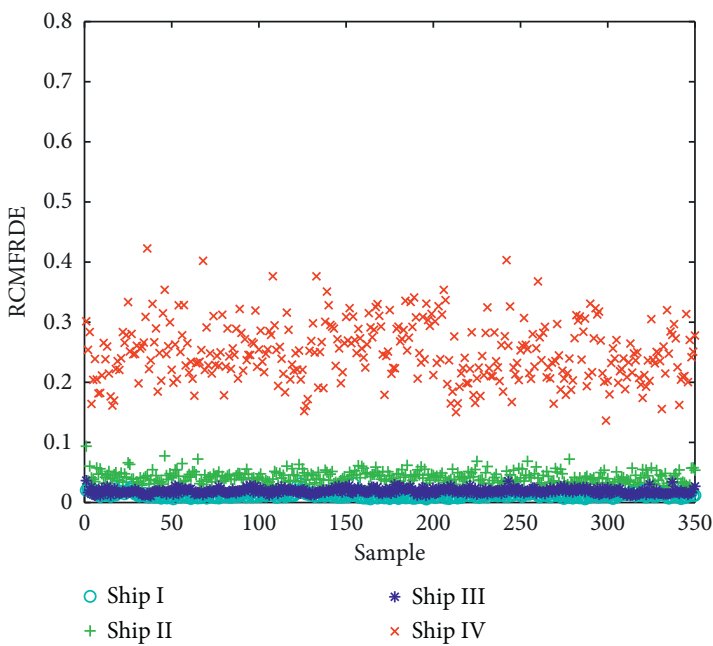

(d)

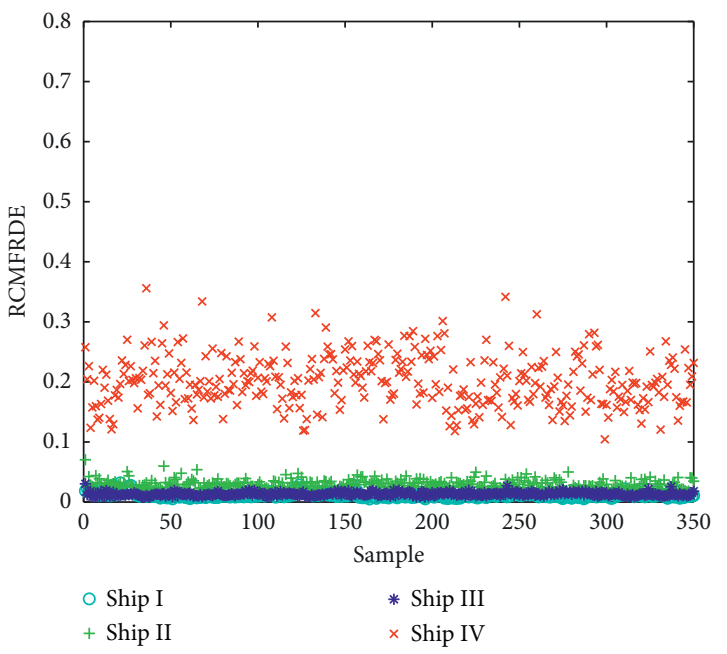

(e)

Figure 3: The distribution of RCMFRDE for four types of S-NS for different scale factors. (a) SF1. (b) SF2. (c) SF3. (d) SF4. (e) SF5. 


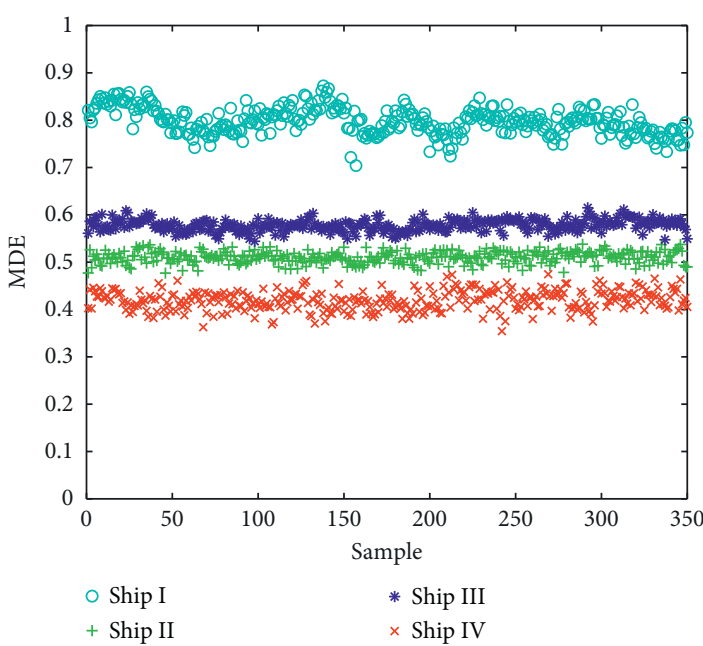

(a)

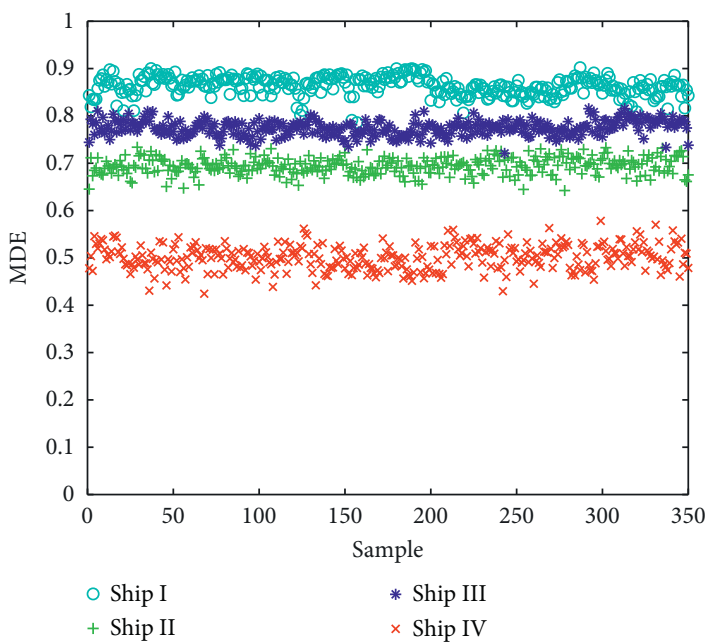

(c)

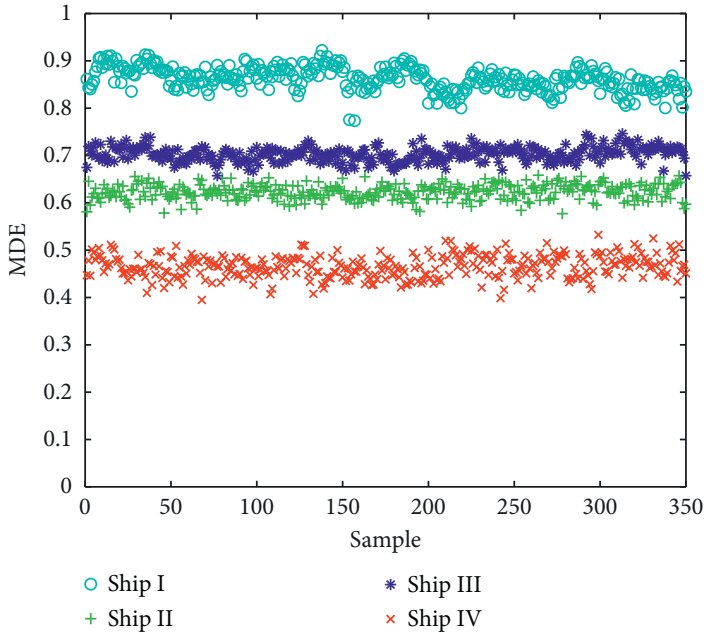

(b)

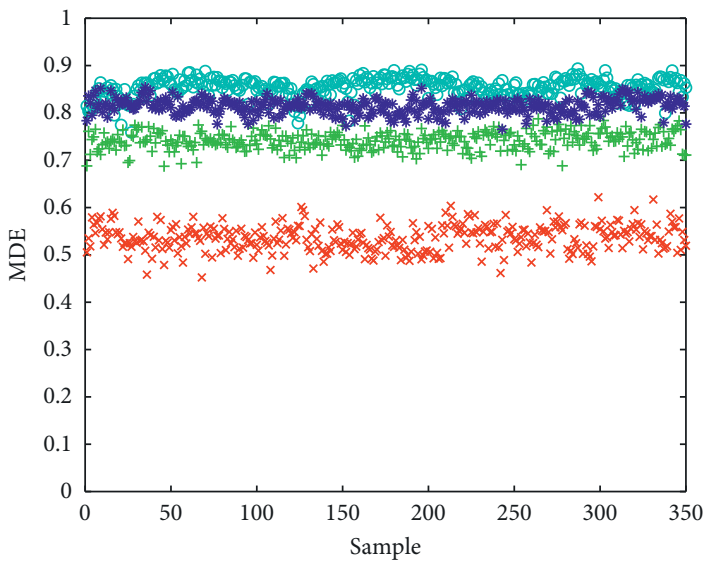

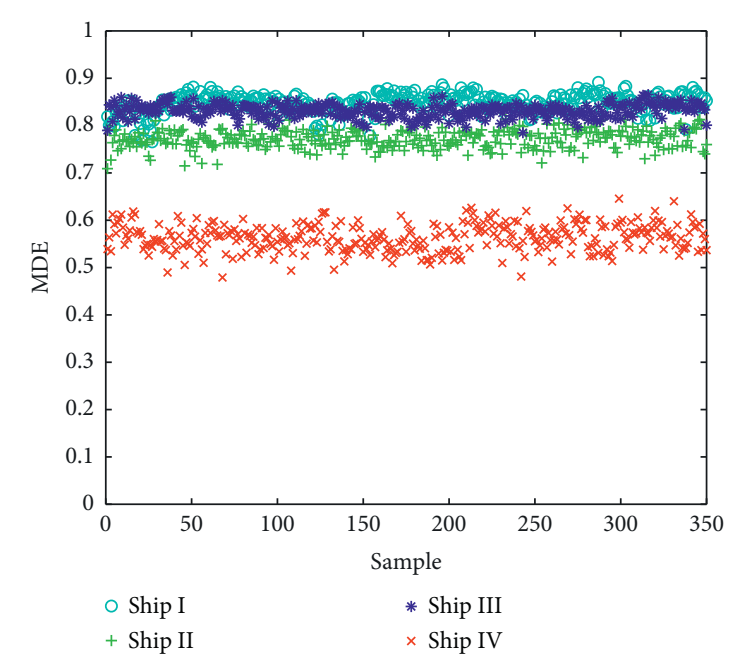

(d)

(e)

Figure 4: The distribution of MDE for four types of S-NS for different scale factors. (a) SF1. (b) SF2. (c) SF3. (d) SF4. (e) SF5. 

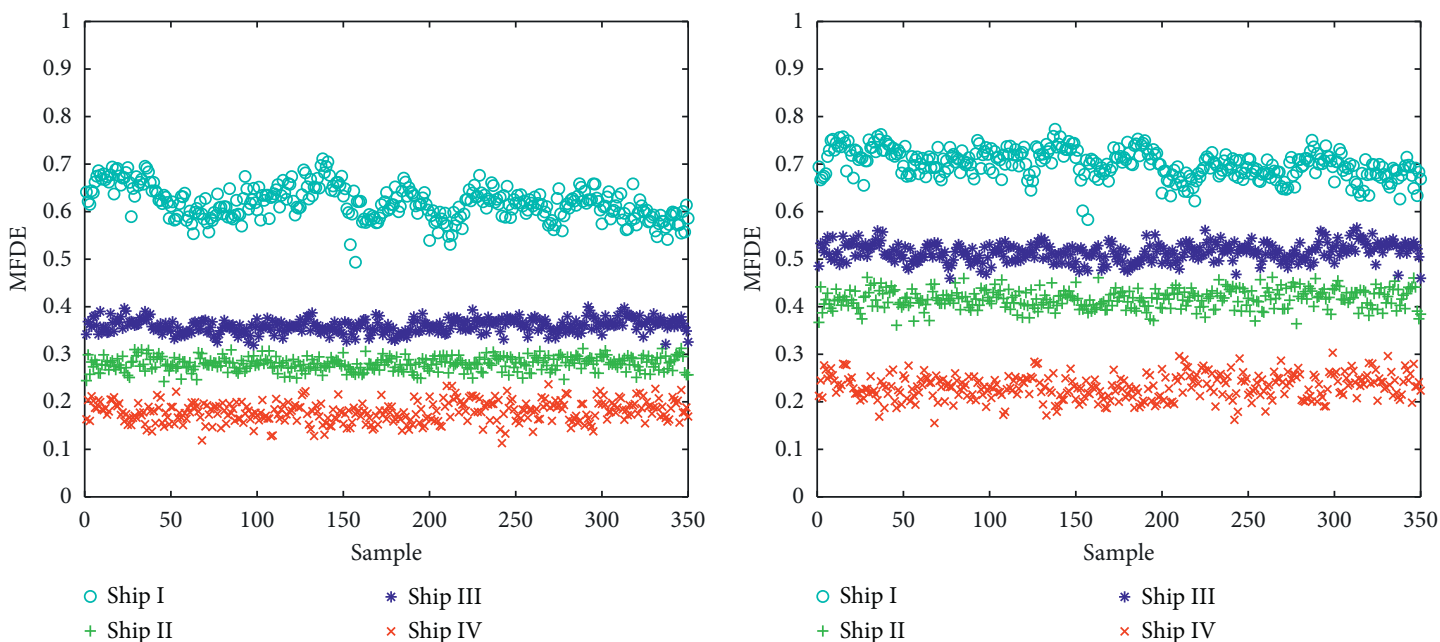

(a)

(b)
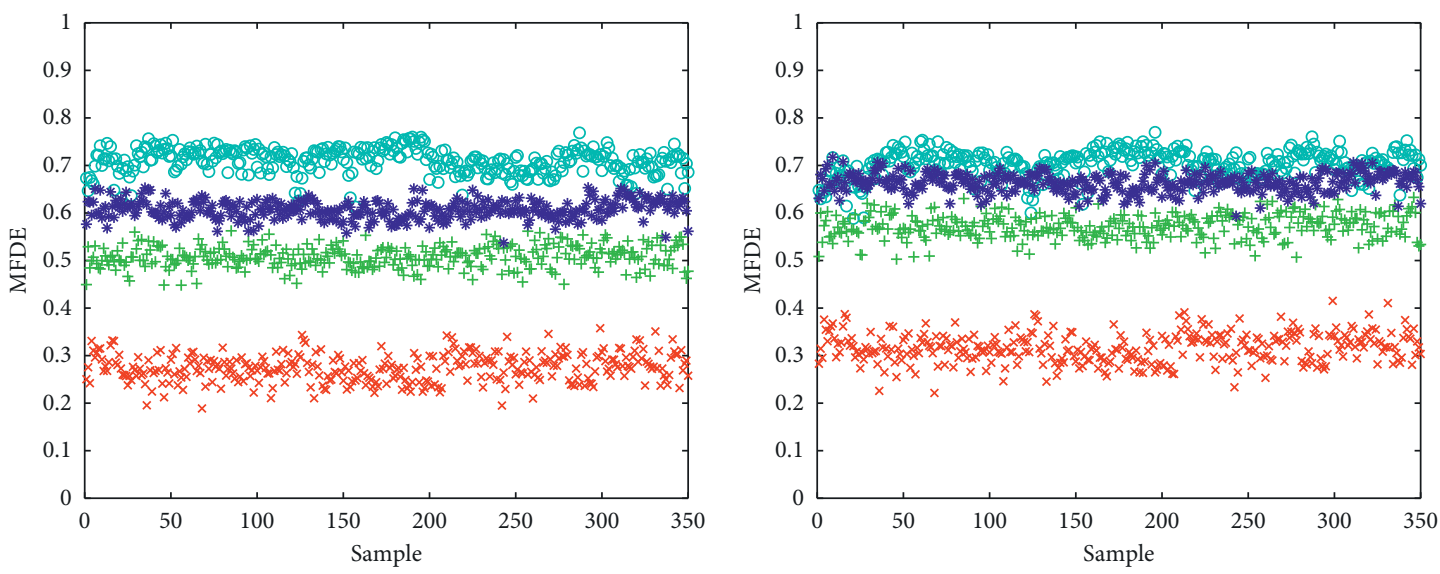

$\begin{array}{ll}\circ \text { Ship I } & * \text { Ship III } \\ + \text { Ship II } & \times \text { Ship IV }\end{array}$

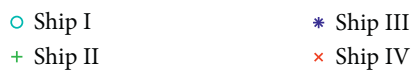

(c)

(d)

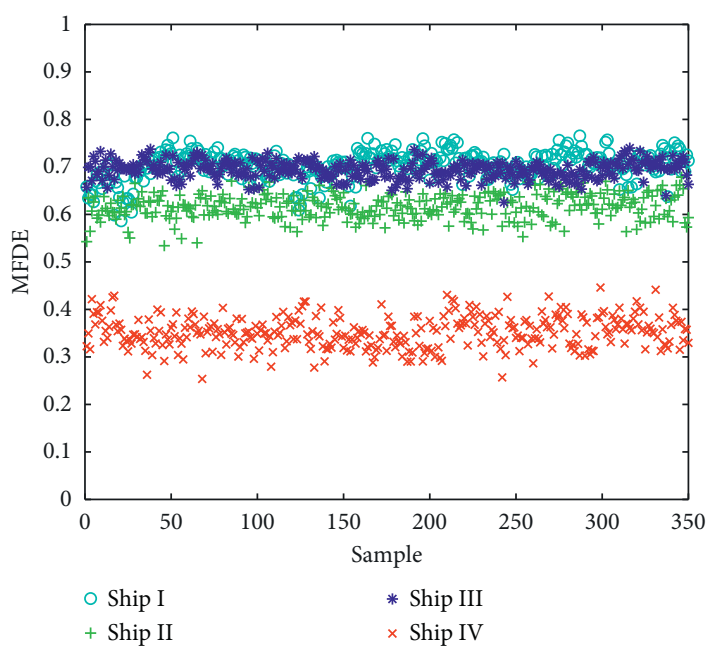

(e)

Figure 5: The distribution of MFDE for four types of S-NS for different scale factors. (a) SF1. (b) SF2. (c) SF3. (d) SF4. (e) SF5. 

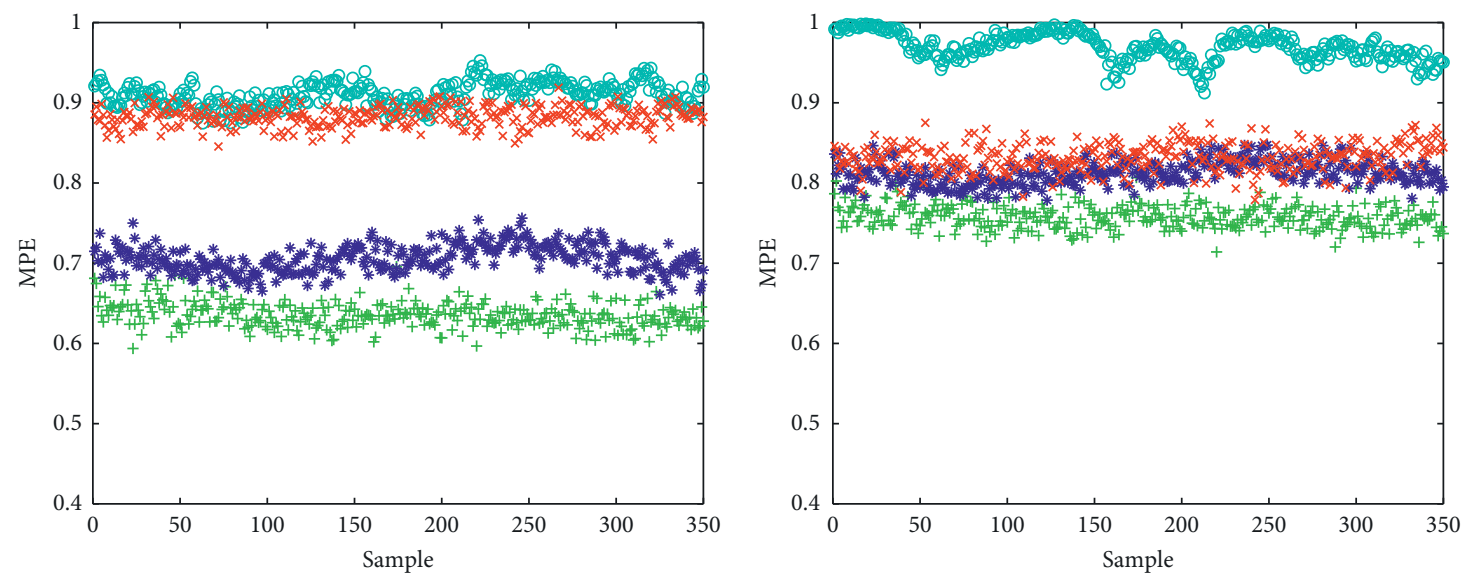

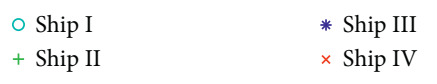

(a)

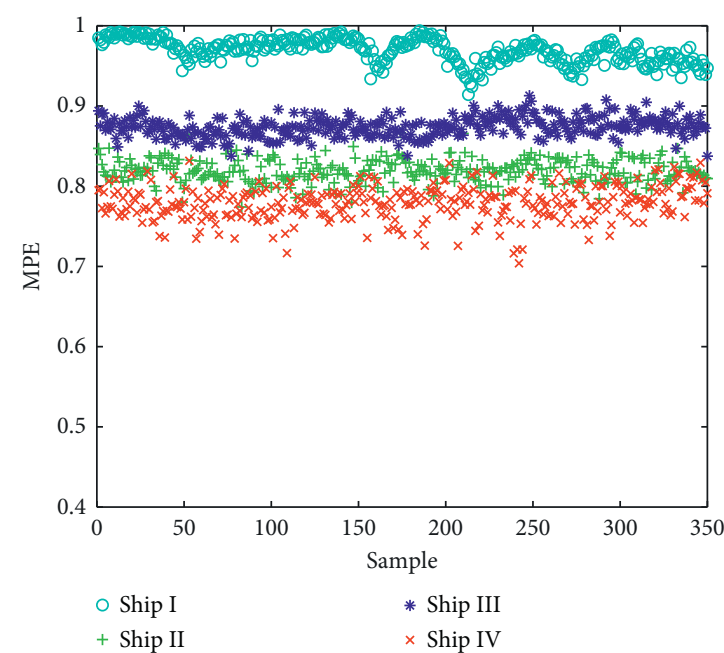

(c)
○ Ship I
* Ship III
+ Ship II
$\times$ Ship IV

(b)

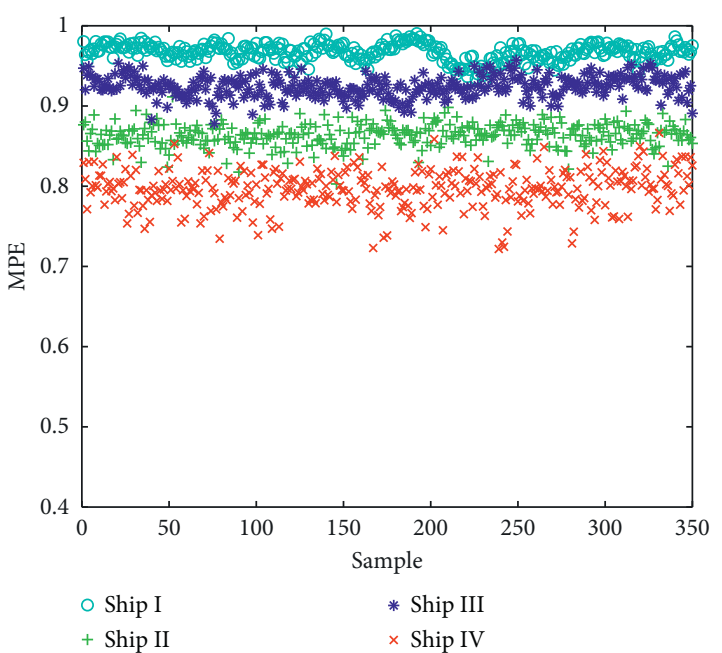

(d)

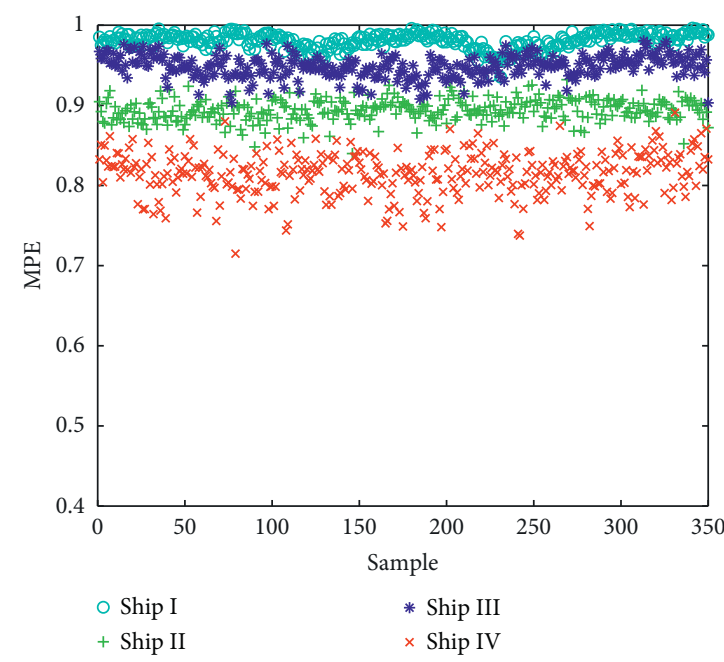

(e)

FIguRE 6: The distribution of MPE for four types of S-NS for different scale factors. (a) SF1. (b) SF2. (c) SF3. (d) SF4. (e) SF5. 


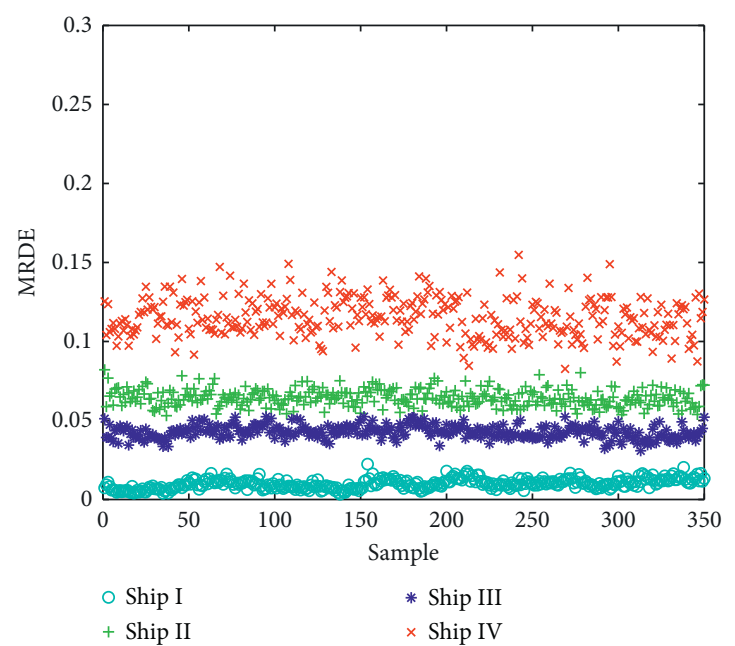

(a)

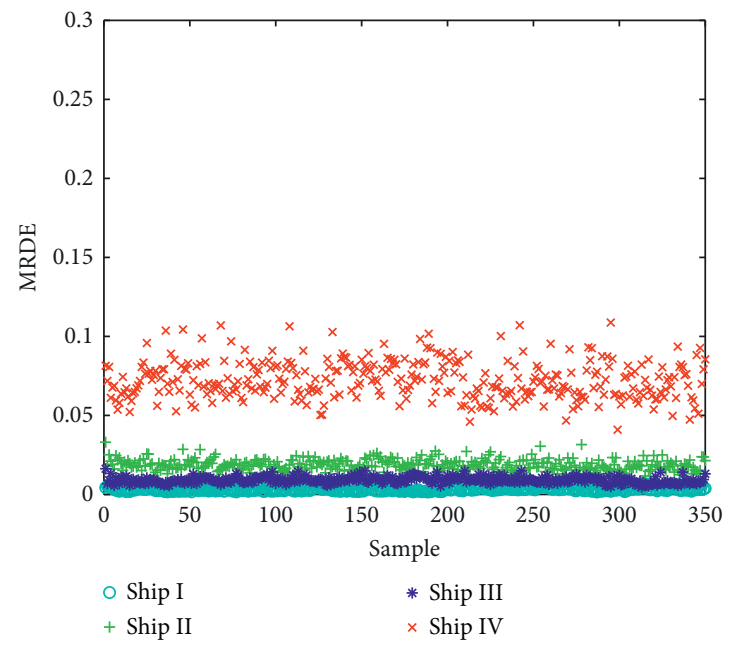

(c)

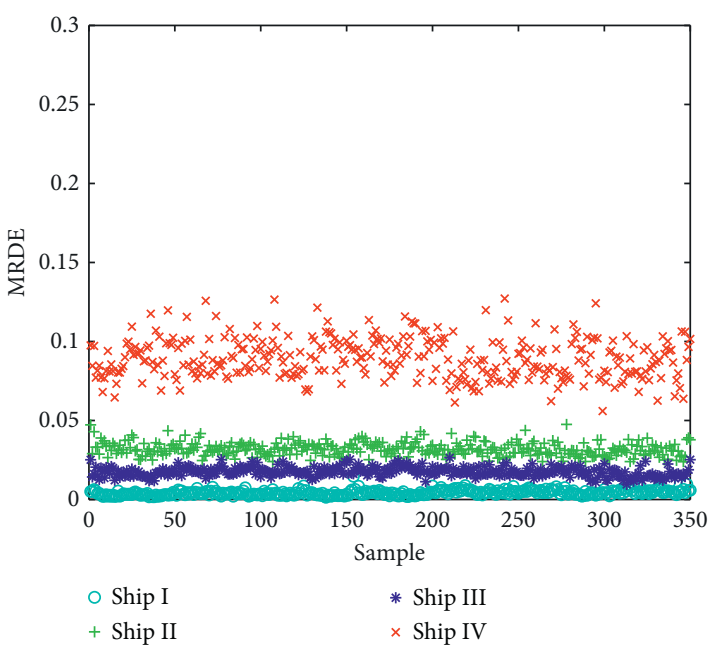

(b)

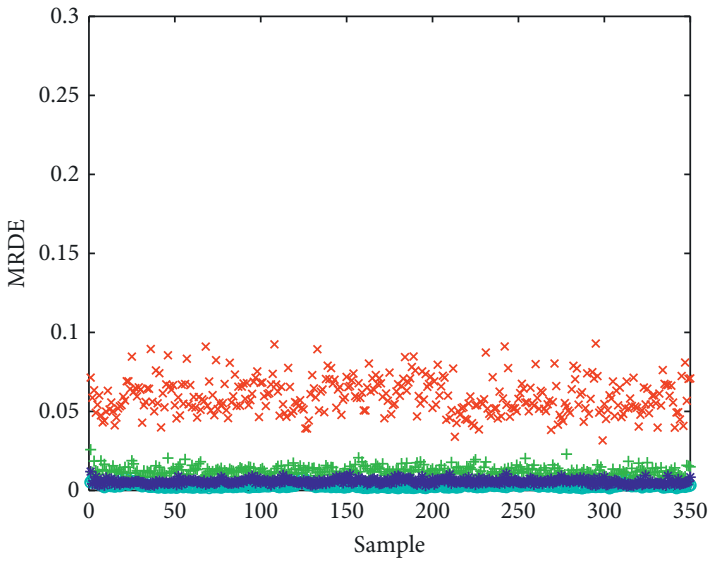
+ Ship II
$\times$ Ship IV

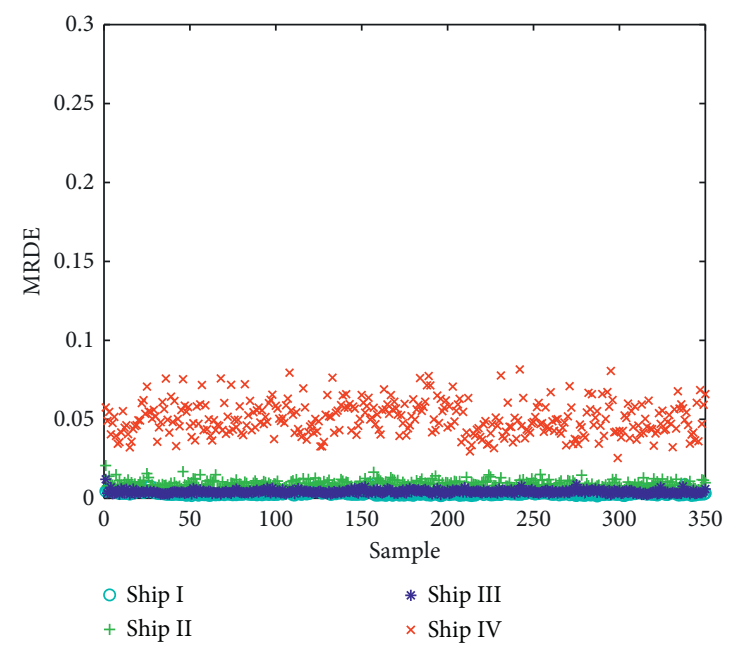

(e)

Figure 7: The distribution of MRDE for four types of S-NS for different scale factors. (a) SF1. (b) SF2. (c) SF3. (d) SF4. (e) SF5. 
TABLe 2: The classification and recognition results of four types of S-NS under single feature.

\begin{tabular}{|c|c|c|c|c|c|c|}
\hline \multirow{2}{*}{ Scale } & \multirow{2}{*}{ Feature } & \multicolumn{4}{|c|}{ Number of incorrectly identified samples } & \multirow{2}{*}{ Average recognition rate (\%) } \\
\hline & & Ship I & Ship II & Ship III & Ship IV & \\
\hline \multirow{5}{*}{1} & RCMFRDE & 0 & 0 & 0 & 3 & 99.75 \\
\hline & MDE & 0 & 0 & 2 & 3 & 99.58 \\
\hline & MFDE & 0 & 0 & 5 & 3 & 99.33 \\
\hline & MPE & 47 & 1 & 24 & 85 & 86.92 \\
\hline & MRDE & 0 & 0 & 5 & 4 & 99.25 \\
\hline \multirow{5}{*}{2} & RCMFRDE & 4 & 10 & 13 & 0 & 97.75 \\
\hline & MDE & 2 & 0 & 2 & 0 & 99.67 \\
\hline & MFDE & 2 & 0 & 7 & 0 & 99.25 \\
\hline & MPE & 0 & 5 & 118 & 108 & 80.75 \\
\hline & MRDE & 5 & 1 & 14 & 0 & 98.33 \\
\hline \multirow{5}{*}{3} & RCMFRDE & 10 & 11 & 26 & 0 & 96.08 \\
\hline & MDE & 5 & 0 & 8 & 0 & 98.92 \\
\hline & MFDE & 8 & 0 & 28 & 0 & 97.00 \\
\hline & MPE & 1 & 70 & 5 & 63 & 88.42 \\
\hline & MRDE & 16 & 12 & 44 & 1 & 93.92 \\
\hline \multirow{5}{*}{4} & RCMFRDE & 80 & 27 & 120 & 0 & 81.08 \\
\hline & MDE & 67 & 14 & 104 & 0 & 84.58 \\
\hline & MFDE & 94 & 17 & 120 & 0 & 80.75 \\
\hline & MPE & 46 & 19 & 7 & 20 & 92.33 \\
\hline & MRDE & 56 & 18 & 129 & 1 & 83.00 \\
\hline \multirow{5}{*}{5} & RCMFRDE & 167 & 59 & 137 & 0 & 69.75 \\
\hline & MDE & 120 & 42 & 129 & 0 & 75.75 \\
\hline & MFDE & 153 & 62 & 147 & 0 & 69.83 \\
\hline & MPE & 68 & 13 & 23 & 6 & 90.83 \\
\hline & MRDE & 109 & 72 & 145 & 1 & 72.75 \\
\hline
\end{tabular}

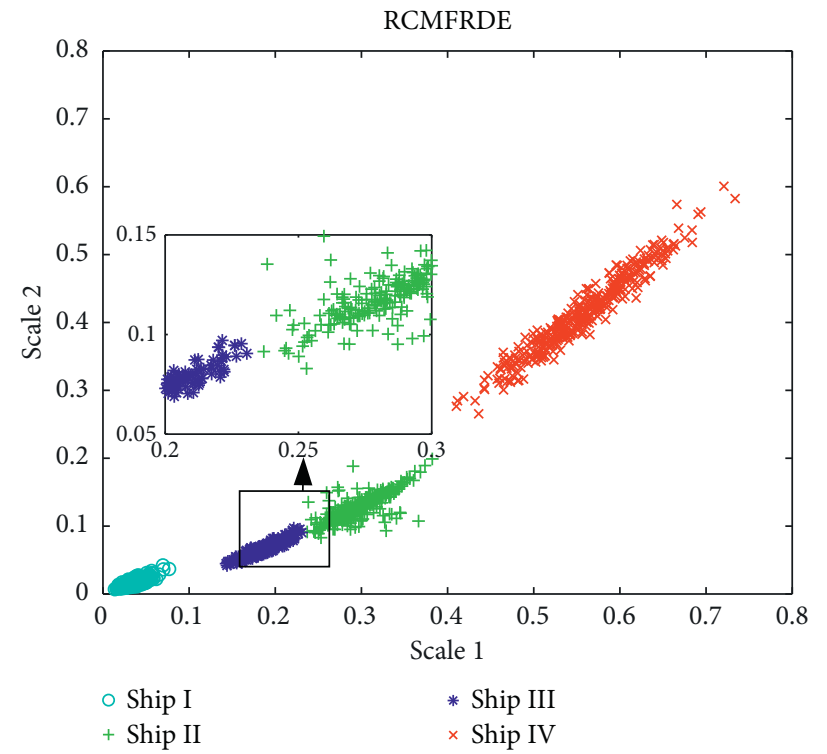

(a)

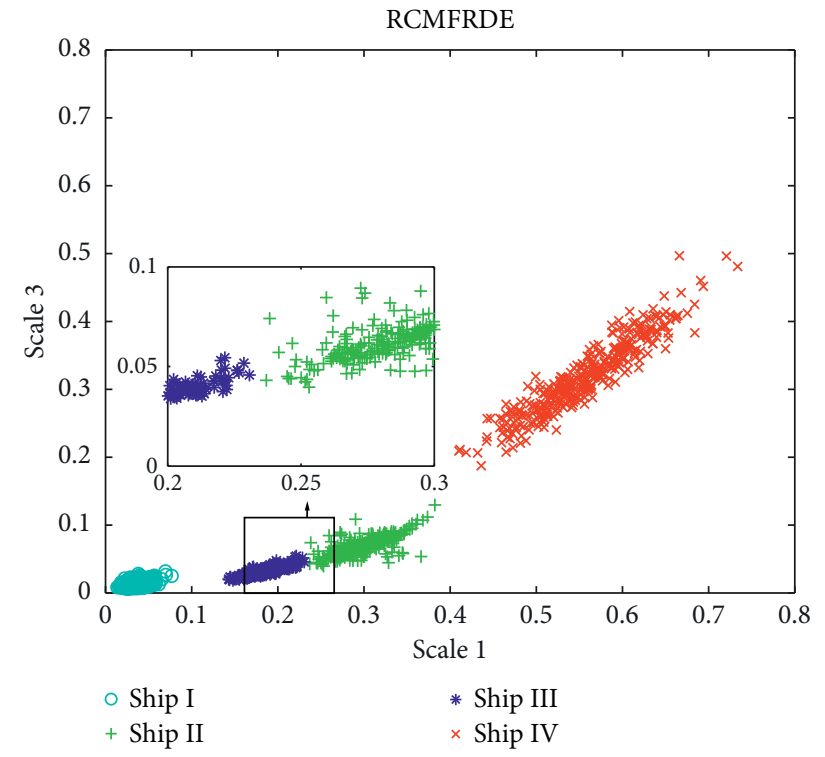

(b)

FIgure 8: Continued. 


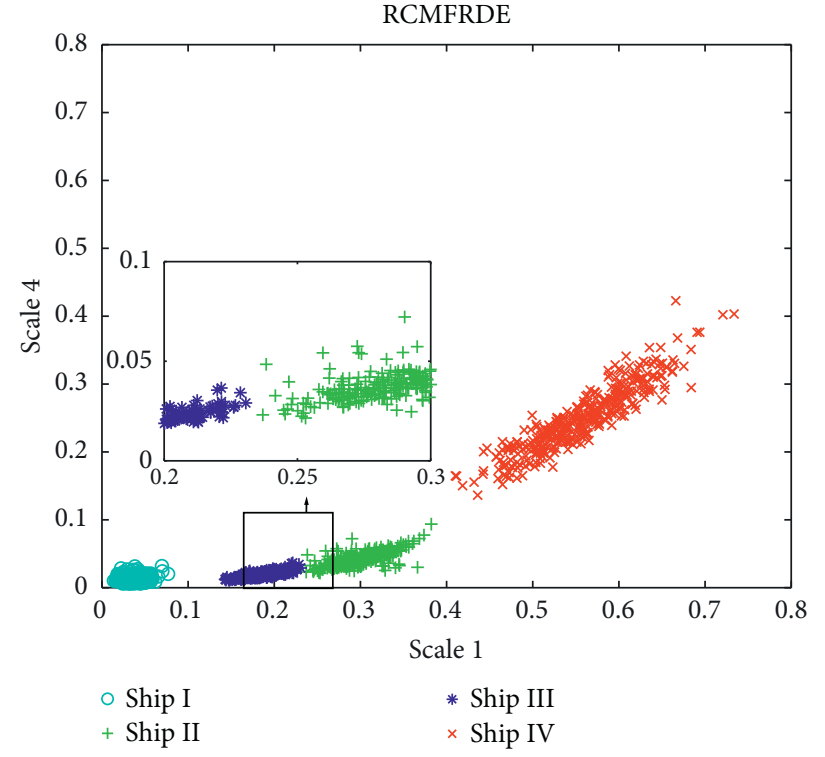

(c)

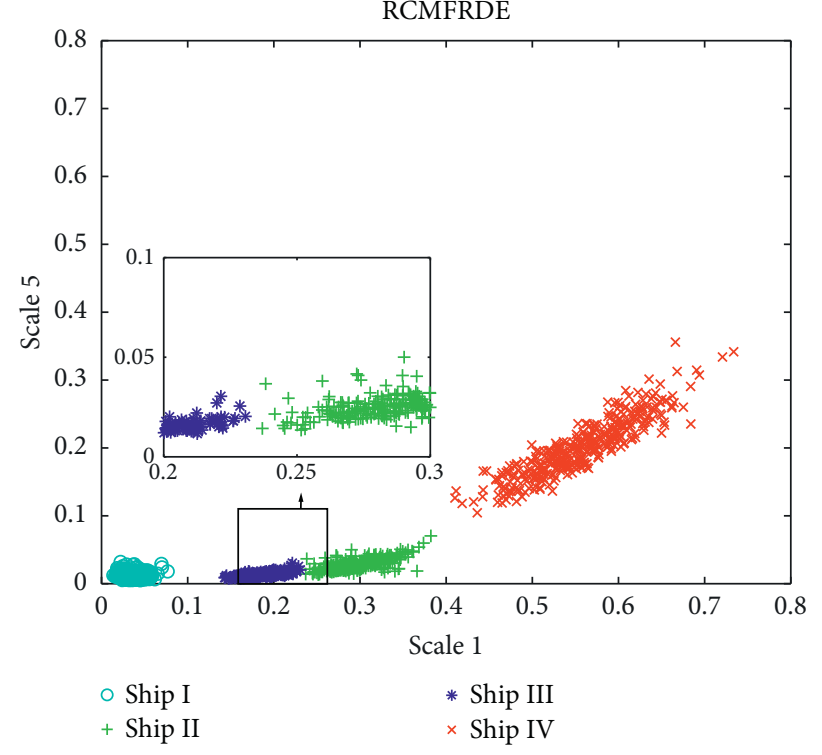

(d)

FiguRE 8: The distribution of RCMFRDE for four types of S-NS under double features. (a) SF1 and SF2. (b) SF1 and SF3. (c) SF1 and SF4. (d) SF1 and SF5.

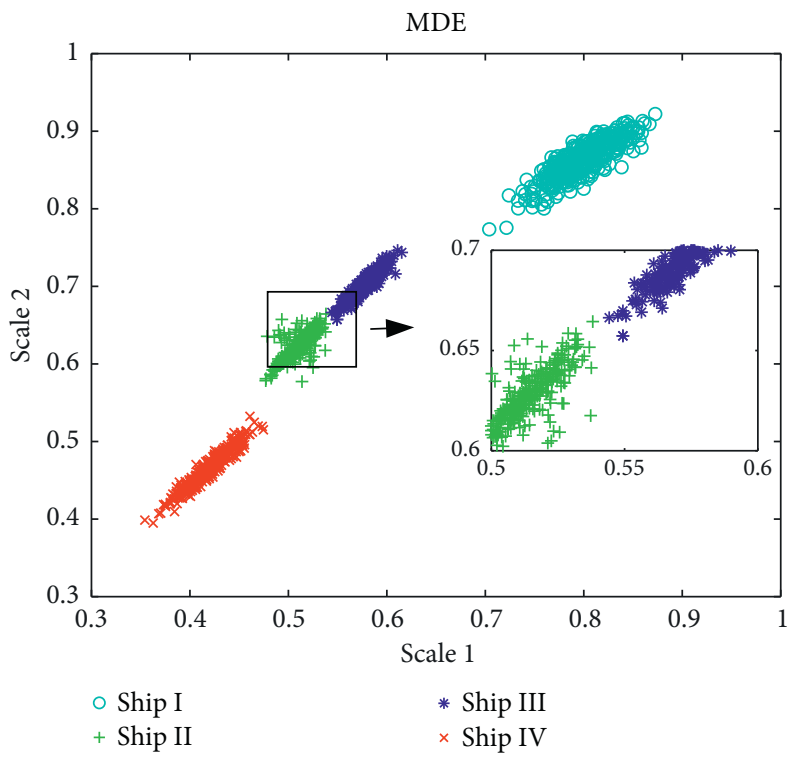

(a)

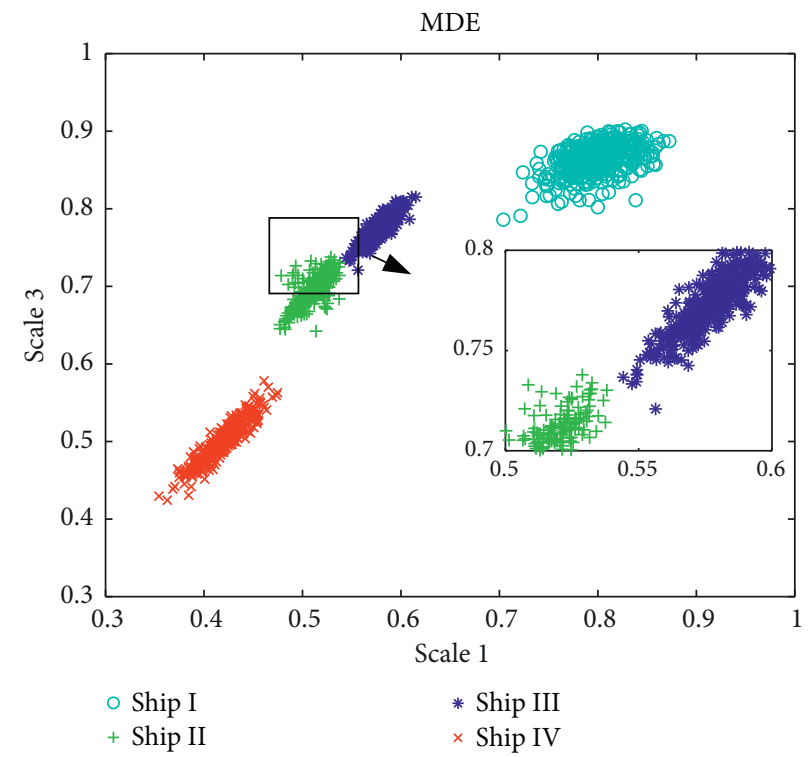

(b)

FIgURE 9: Continued. 


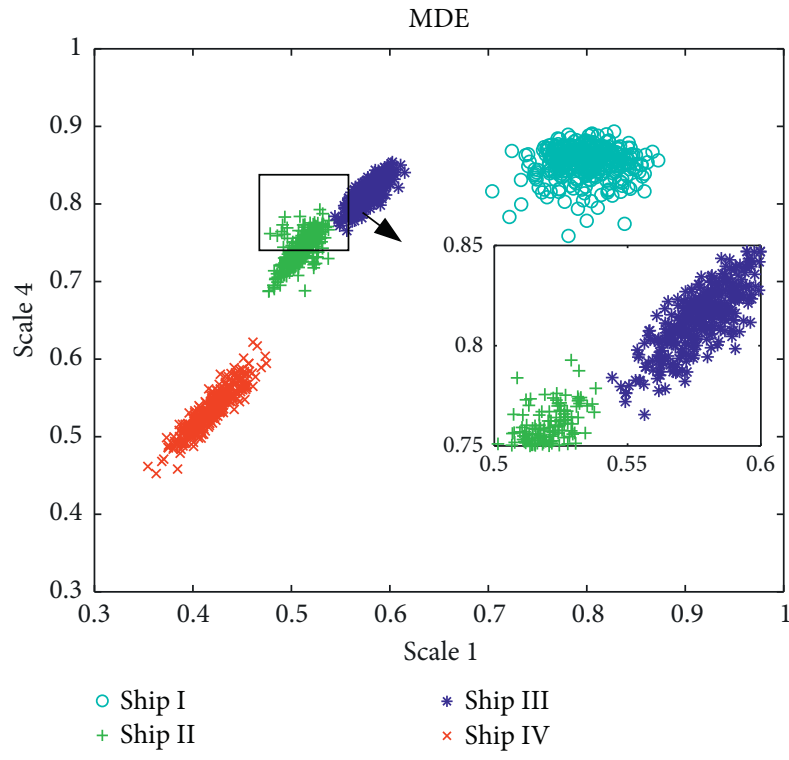

(c)

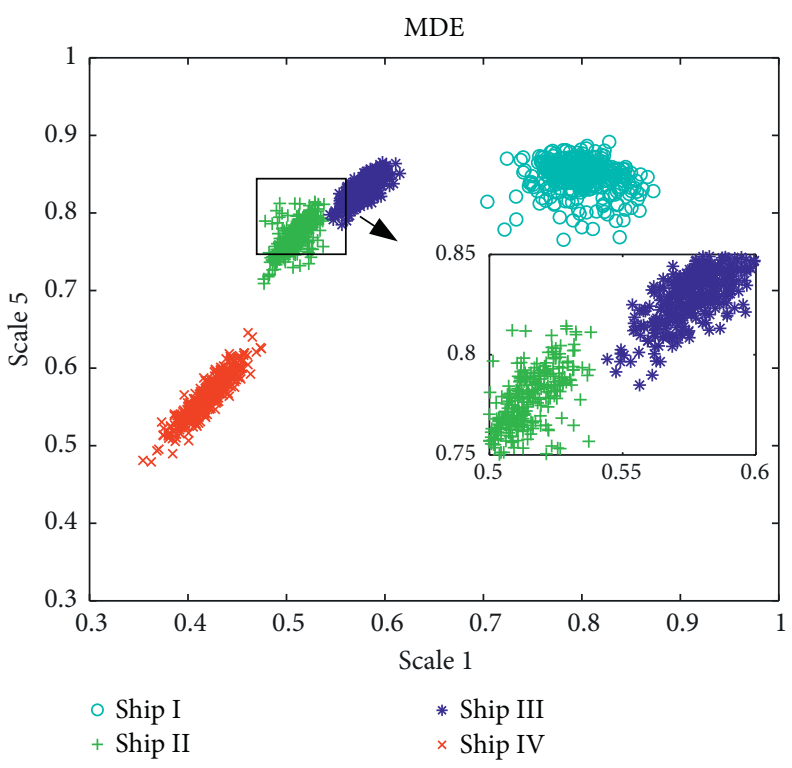

(d)

Figure 9: The distribution of MDE for four types of S-NS under double features. (a) SF1 and SF2. (b) SF1 and SF3. (c) SF1 and SF4. (d) SF1 and SF5.

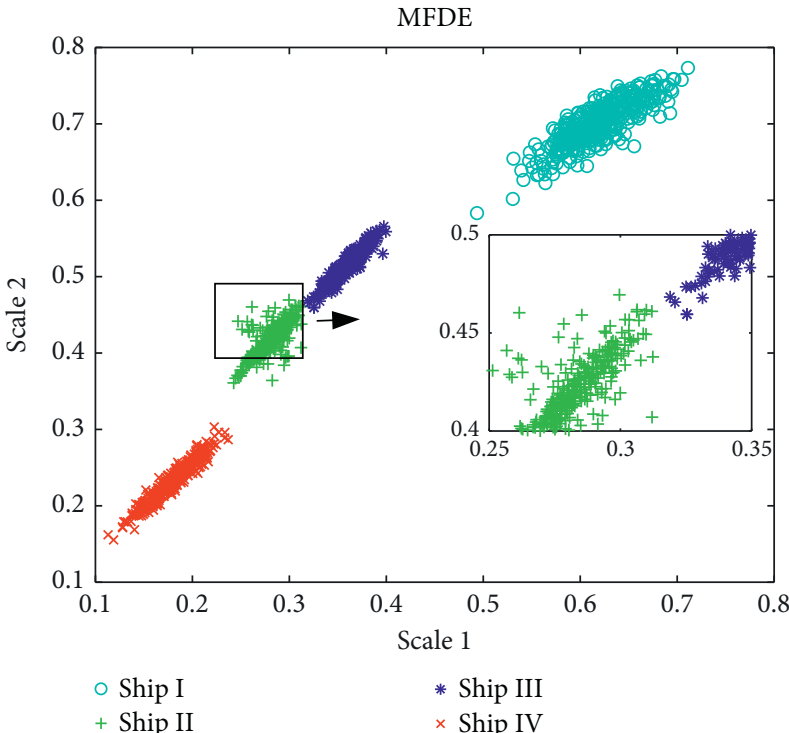

(a)

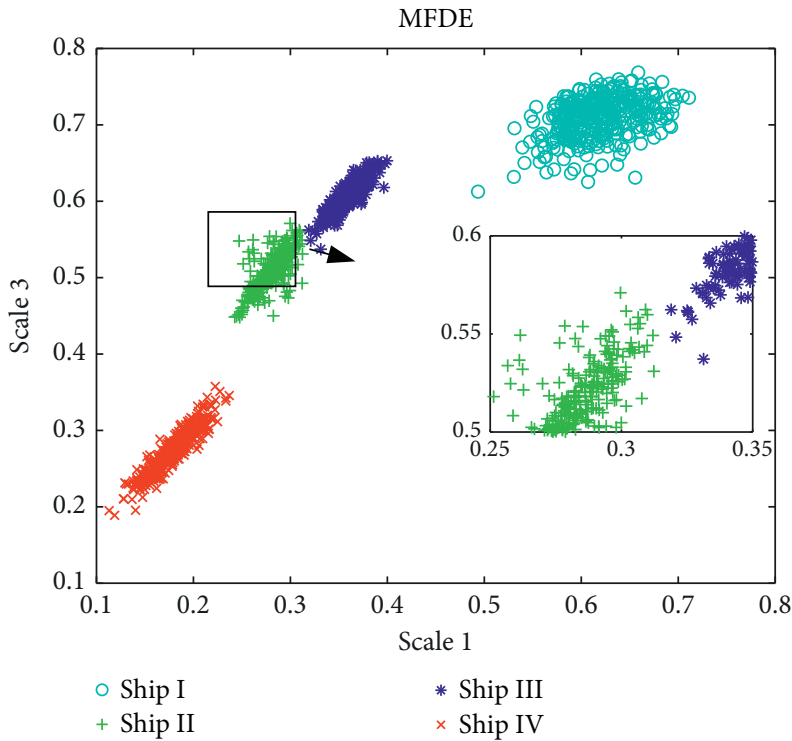

(b)

Figure 10: Continued. 


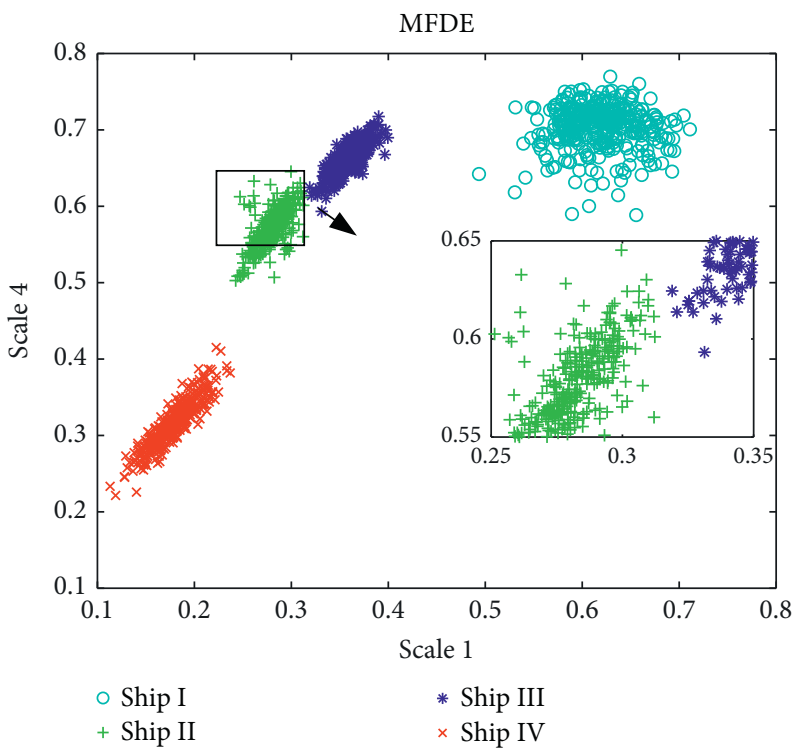

(c)
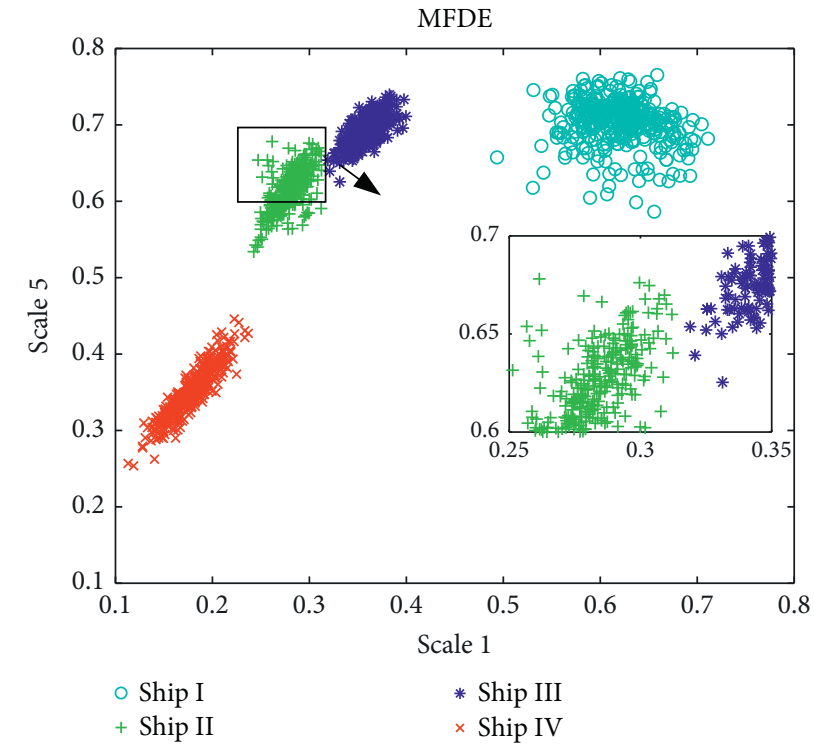

(d)

FIGURE 10: The distribution of MFDE for four types of S-NS under double features. (a) SF1 and SF2. (b) SF1 and SF3. (c) SF1 and SF4. (d) SF1 and SF5.

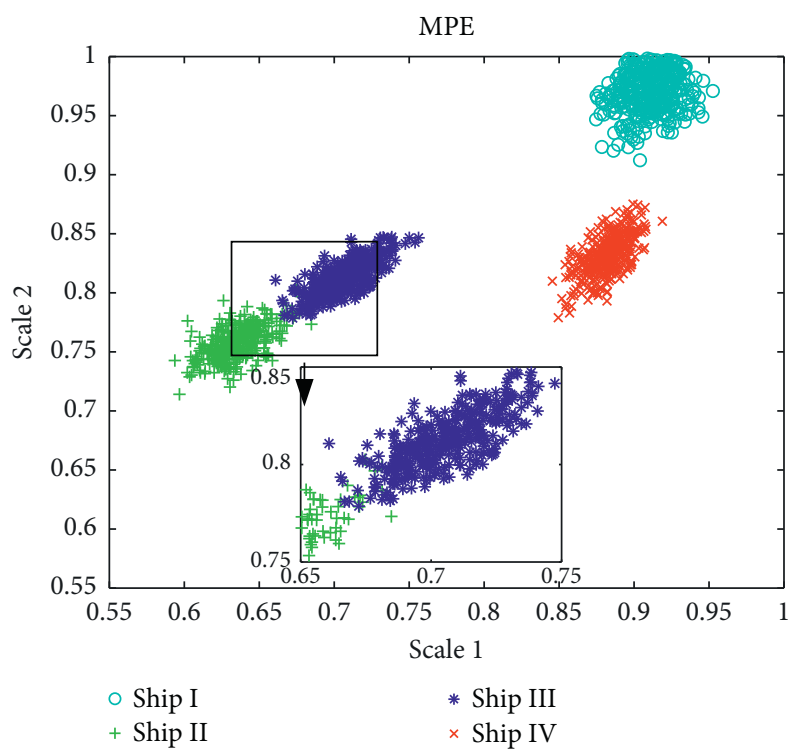

(a)

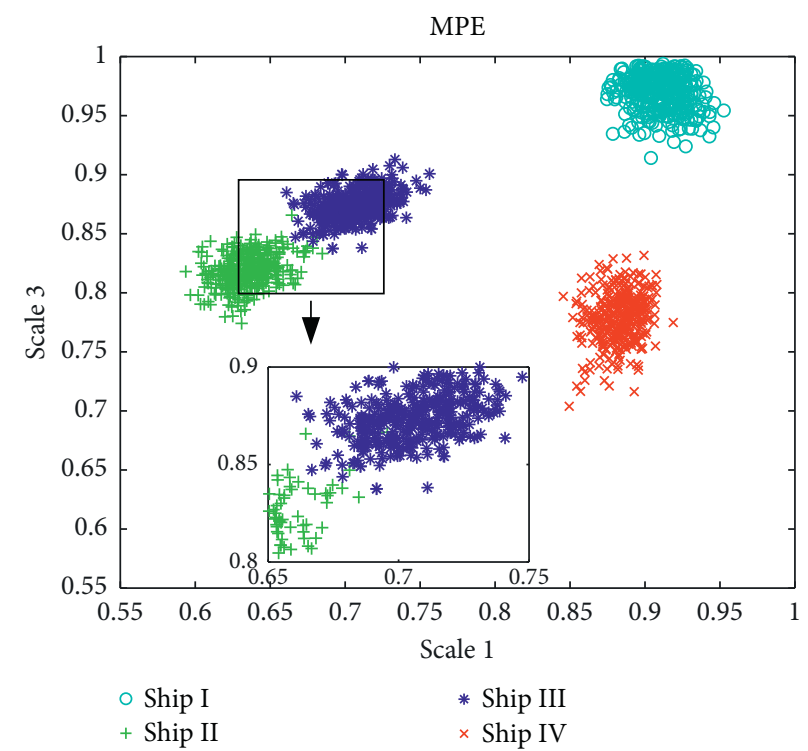

(b)

FIgURE 11: Continued. 


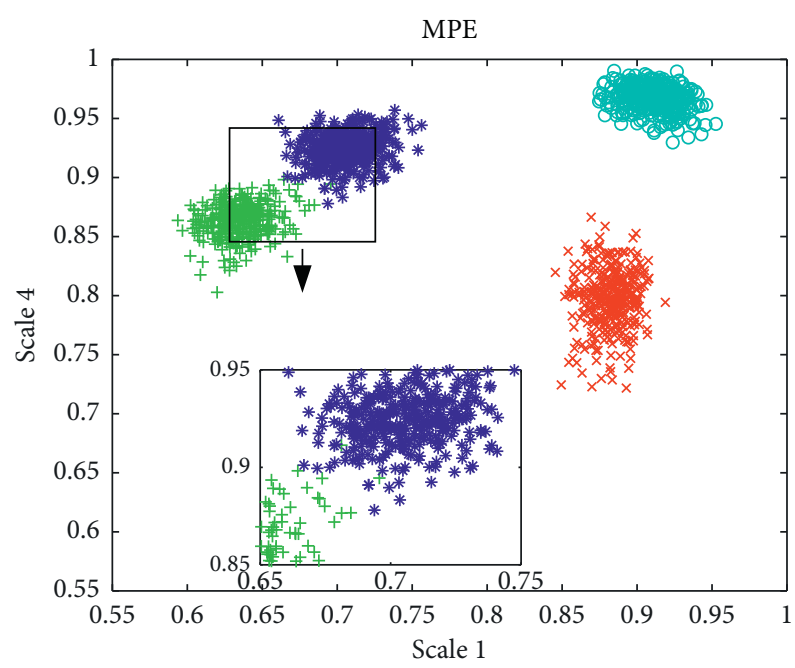

$\begin{array}{ll}\circ \text { Ship I } & * \text { Ship III } \\ + \text { Ship II } & \times \text { Ship IV }\end{array}$

(c)

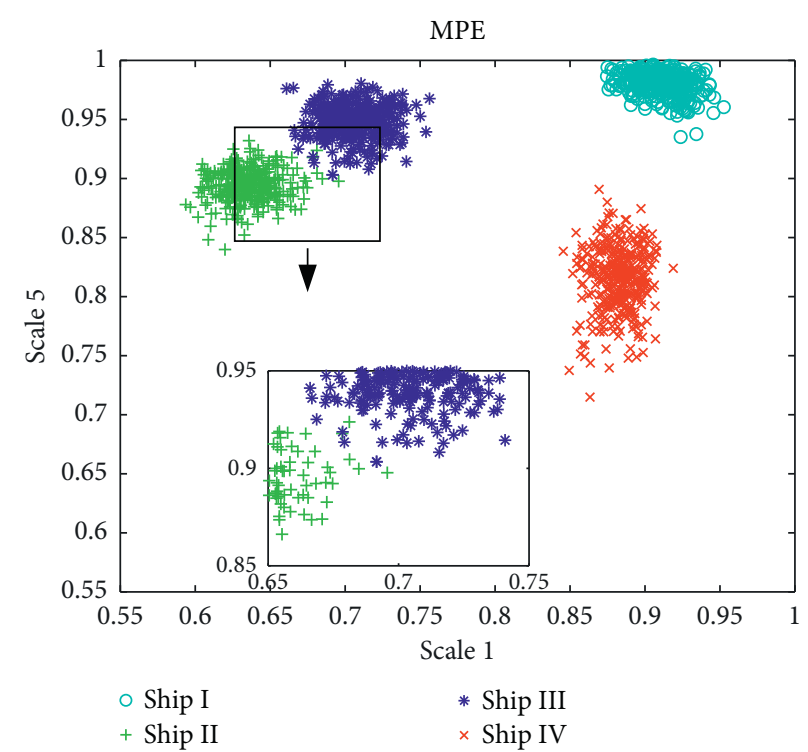

(d)

FIGURE 11: The distribution of MPE for four types of S-NS under double features. (a) SF1 and SF2. (b) SF1 and SF3. (c) SF1 and SF4. (d) SF1 and SF5.

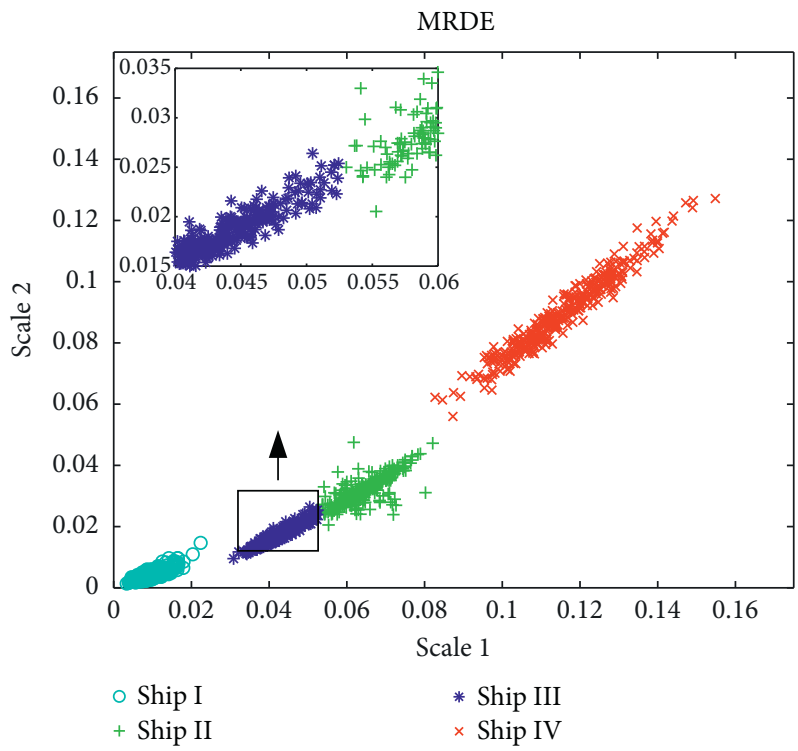

(a)

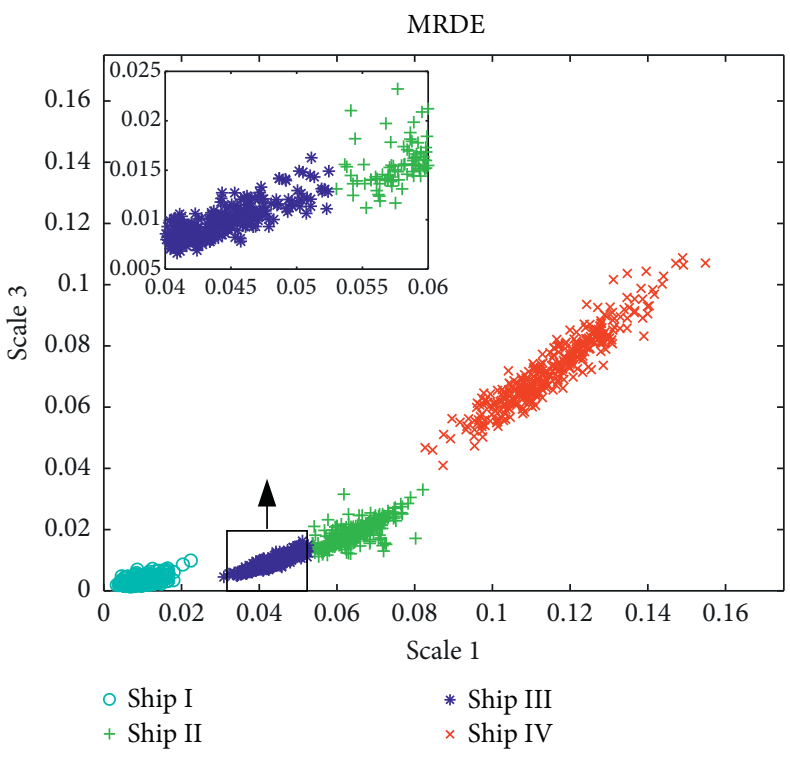

(b)

FIgUre 12: Continued. 


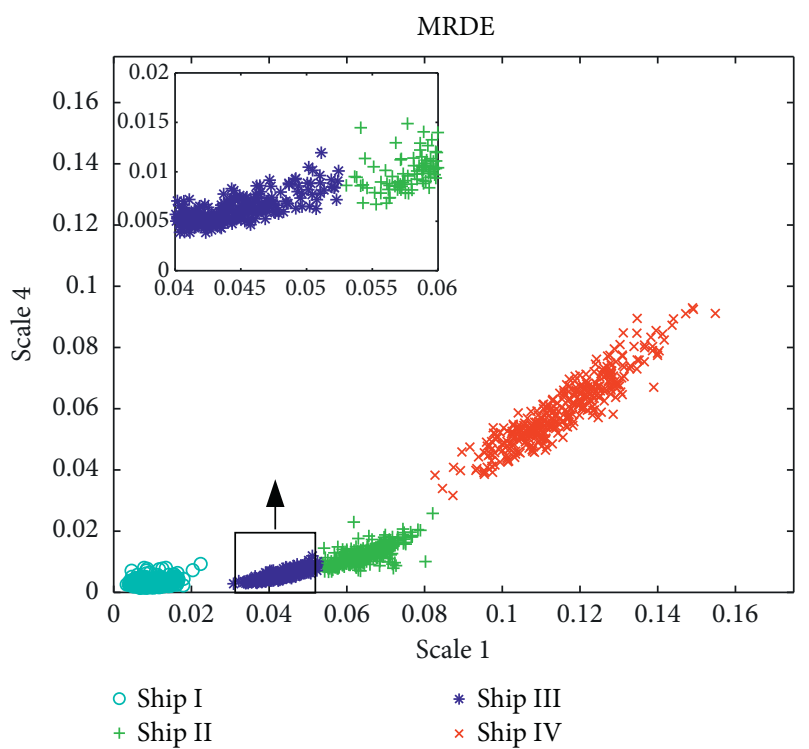

(c)

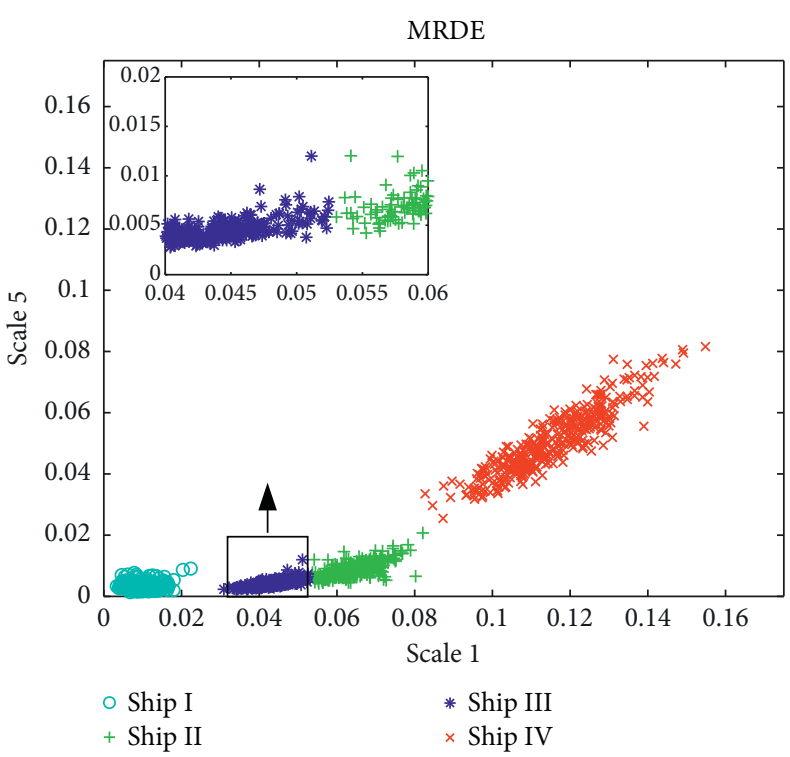

(d)

FIGURE 12: The distribution of MRDE for four types of S-NS under double features. (a) SF1 and SF2. (b) SF1 and SF3. (c) SF1 and SF4. (d) SF1 and SF5.

TABLE 3: The classification and recognition results of four types of S-NS under double features.

\begin{tabular}{|c|c|c|c|c|c|c|}
\hline \multirow{2}{*}{ Scales } & \multirow{2}{*}{ Feature } & \multicolumn{4}{|c|}{ Number of incorrectly identified samples } & \multirow{2}{*}{ Average recognition rate $(\%)$} \\
\hline & & Ship I & Ship II & Ship III & Ship IV & \\
\hline \multirow{5}{*}{1 and 2} & RCMFRDE & 0 & 0 & 0 & 0 & 100.00 \\
\hline & MDE & 0 & 0 & 3 & 0 & 99.75 \\
\hline & MFDE & 1 & 0 & 5 & 0 & 99.50 \\
\hline & MPE & 0 & 1 & 20 & 0 & 98.25 \\
\hline & MRDE & 1 & 0 & 6 & 1 & 99.33 \\
\hline \multirow{5}{*}{1 and 3} & RCMFRDE & 0 & 0 & 0 & 0 & 100.00 \\
\hline & MDE & 0 & 0 & 3 & 0 & 99.75 \\
\hline & MFDE & 1 & 0 & 7 & 0 & 99.33 \\
\hline & MPE & 0 & 3 & 7 & 0 & 99.17 \\
\hline & MRDE & 1 & 1 & 14 & 1 & 98.58 \\
\hline \multirow{5}{*}{1 and 4} & RCMFRDE & 0 & 0 & 0 & 0 & 100.00 \\
\hline & MDE & 0 & 0 & 4 & 0 & 99.67 \\
\hline & MFDE & 1 & 0 & 7 & 0 & 99.33 \\
\hline & MPE & 0 & 2 & 6 & 0 & 99.33 \\
\hline & MRDE & 0 & 1 & 16 & 2 & 98.42 \\
\hline \multirow{5}{*}{1 and 5} & RCMFRDE & 0 & 0 & 0 & 0 & 100.00 \\
\hline & MDE & 0 & 0 & 3 & 0 & 99.75 \\
\hline & MFDE & 1 & 0 & 6 & 0 & 99.42 \\
\hline & MPE & 0 & 0 & 5 & 0 & 99.58 \\
\hline & MRDE & 0 & 1 & 18 & 1 & 98.33 \\
\hline
\end{tabular}

based feature extraction method combined with two scales has the highest recognition rate of S-NS up to $100 \%$.

In summary, compared with the single scale condition, the average recognition rate of RCMFRDE is improved, utilizing $13.05 \%$. The double feature extraction method of RCMFRDE for S-NS can fully recognize four types of S-NS.

\section{Conclusions}

In this paper, RCMFRDE is proposed on the basis of FRDE and applied to S-NS feature extraction. The feasibility of the proposed method is verified through feature extraction and classification recognition experiments on four types of measured S-NS, and the main conclusions are drawn as follows:

(1) Based on the theory of DE, this paper introduces the operation of refined composite by combining the advantages of RDE and MFDE and proposes RCMFRDE for S-NS feature extraction.

(2) Compared with the other four single feature extraction methods, the recognition rate of the RCMFRDE-based single feature extraction method 
proposed in this paper is the highest and reaches $99.75 \%$, which is $10.19 \%$ higher than the other average recognition rates.

(3) The proposed double feature extraction method further improves the classification performance of the single feature extraction method. Compared with the other four double feature extraction methods, the RCMFRDE-based double feature extraction method has the best recognition of the four types of S-NS with a recognition rate of $100 \%$.

\section{Data Availability}

The data used to support the findings of this study are available from the corresponding author upon request.

\section{Conflicts of Interest}

The authors declare that they have no conflicts of interest.

\section{References}

[1] J. D. Tucker and M. R. Azimi-Sadjadi, "Coherence-based underwater target detection from multiple disparate sonar platforms," IEEE Journal of Oceanic Engineering, vol. 36, no. 1, pp. 37-51, 2011.

[2] S. Siddagangaiah, Y. Li, X. Guo, and K. Yang, "On the dynamics of ocean ambient noise: two decades later," Chaos: An Interdisciplinary Journal of Nonlinear Science, vol. 25, no. 10, Article ID 103117, 2015.

[3] S. Wang and X. Zeng, "Robust underwater noise targets classification using auditory inspired time-frequency analysis," Applied Acoustics, vol. 78, no. 4, pp. 68-76, 2014.

[4] J. Guo, X. Liu, S. Li, and Z. Wang, "Bearing Intelligent fault diagnosis based on wavelet transform and convolutional neural network," Shock and Vibration, vol. 2020, no. 19, 14 pages, Article ID 6380486, 2020.

[5] S. D. Lu, H. W. Sian, M. H. Wang, and C. C. Kuo, "Fault diagnosis of power capacitors using a convolutional neural network combined with the chaotic synchronisation method and the empirical mode decomposition method," IET Science, Measurement \& Technology, vol. 15, no. 7, pp. 551-561, 2021.

[6] Y. Zhang, Z. Lian, W. Fu, and X. Chen, "An ESR quasi-online identification method for the fractional-order capacitor of forward converters based on variational mode decomposition," IEEE Transactions on Power Electronics, p. 1, 2021.

[7] S. Jiao, B. Geng, Y. Li, Q. Zhang, and Q. Wang, "Fluctuationbased reverse dispersion entropy and its applications to signal classification," Applied Acoustics, vol. 175, no. 4, Article ID 107857, 2021.

[8] Y. Li, S. Jiao, and B. Geng, "A comparative study of four multiscale entropies combined with grey relational degree in classification of ship-radiated noise," Applied Acoustics, vol. 176, no. 4, Article ID 107865, 2021.

[9] S. Tucker and G. J Brown, "Classification of transient sonar sounds using perceptually motivated features[J]," IEEE Journal of Ocean Engineering, vol. 30, no. 3, pp. 588-600, 2011.

[10] R. Pascoal and C. Guedes Soares, "Non-parametric wave spectral estimation using vessel motions," Applied Ocean Research, vol. 30, no. 1, pp. 46-53, 2008.

[11] Y. Guo, W. Rao, and Y. Han, "Extraction of higher-order coupling feature using three and one half dimension spectrum," Applied Mathematics and Computation, vol. 185, no. 2, pp. 798-809, 2007.

[12] Jović, A. Bogunović, and Nikola, "Feature extraction for ECG time-series mining based on chaos theory," in Proceedings of the International Conference on Information Technology Interfaces, no. 2, pp. 64-68, Article ID IEEE, Cavtat, Croatia, June 2007.

[13] C. Ioana, A. Quinquis, and Y. Stephan, "Feature extraction from underwater signals using time-frequency warping operators," IEEE Journal of Oceanic Engineering, vol. 31, no. 3, pp. 628-645, 2006.

[14] D. Bruzzone, C. Gironi, and A. Grasso, "Nonlinear effects on motions and loads using an iterative time-frequency solver," International Journal of Naval Architecture and Ocean Engineering, vol. 3, no. 1, pp. 20-26, 2011.

[15] B. Yu, Y. H. Li, and P. Zhang, "Application of correlation dimension and kolmogorov entropy in aeroengine fault diagnosis," Journal of Aerospace Power, vol. 21, no. 1, pp. 219-224, 2006.

[16] P. Grassberger and I. Procaccia, "Characterization of strange attractors," Physical Review Letters, vol. 50, no. 5, pp. 346-349, 1983.

[17] H. J. Shyu and R Hillson, "Integrating ocean acoustic propagation models and marine mammal auditory models," in Proceedings of the Ocean's 2002 Conference and Exhibition, Marine Technology Society, IEEE, OES, Institute of Electrical and Electronics Engineers Inc., Jackson, MI, USA, October 2002.

[18] A. Lempel and J. Ziv, "On the complexity of finite sequences," IEEE Transactions on Information Theory, vol. 22, no. 1, pp. 75-81, 1976.

[19] L. Cui, X. Gong, J. Zhang, and H. Wang, "Double-dictionary matching pursuit for fault extent evaluation of rolling bearing based on the lempel-ziv complexity," Journal of Sound and Vibration, vol. 385, pp. 372-388, 2016.

[20] M. Kedadouche, T. Kidar, M. Thomas, A. Tahan, M El Badaoui, and R. Guilbault, "Combining EMD and lempel-ziv complexity for early detection of gear cracks," Proceedings of the International Conference Surveillance, vol. 7, pp. 100-10, 2013.

[21] D. Abásolo, R. Hornero, C. Gómez, M. García, and M. López, “Analysis of EEG background activity in alzheimer's disease patients with lempel-ziv complexity and central tendency measure," Medical Engineering \& Physics, vol. 28, no. 4, pp. 315-322, 2006.

[22] M. Aboy, R. Hornero, D. Abasolo, and D. Alvarez, "Interpretation of the lempel-ziv complexity measure in the context of biomedical signal analysis," IEEE Transactions on Biomedical Engineering, vol. 53, no. 11, pp. 2282-2288, 2006.

[23] D. Z. Zhang, "Research on the correlation between the mutual information and lempel-ziv complexity of nonlinear time series," Acta Physica Sinica -Chinese Edition, vol. 56, no. 6, 2007.

[24] X. Mao, P. Shang, M. Xu, and C. K. Meng, "Measuring time series based on multiscale dispersion lempel-ziv complexity and dispersion entropy plane," Chaos, Solitons \& Fractals, vol. 137, Article ID 109868, 2020.

[25] Y. Bai, Z. Liang, and X. Li, "A permutation lempel-ziv complexity measure for EEG analysis," Biomedical Signal Processing and Control, vol. 19, pp. 102-114, 2015.

[26] C. E. Shannon, "A mathematical theory of communication," Bell System Technical Journal, vol. 27, no. 4, pp. 623-656, 1948. 
[27] C. Bandt and B. Pompe, "Permutation entropy: a natural complexity measure for time series," Physical Review Letters, vol. 88, no. 17, Article ID 174102, 2002.

[28] B. Fadlallah, B. Chen, A. Keil, and J. Principe, "Weightedpermutation entropy: a complexity measure for time series incorporating amplitude information," Physical Review E Statistical Nonlinear \& Soft Matter Physics, vol. 87, no. 2, Article ID 022911, 2013.

[29] Y. Li, Y. Li, X. Chen, and Y. Jing, "A novel feature extraction method for ship-radiated noise based on variational mode decomposition and multi-scale permutation entropy," Entropy, vol. 19, no. 7, 2017.

[30] H. Azami and J. Escudero, "Amplitude-aware permutation entropy: illustration in spike detection and signal segmentation," Computer Methods and Programs in Biomedicine, vol. 128, pp. 40-51, 2016.

[31] M. Rostaghi and H. Azami, "Dispersion entropy: a measure for time-series analysis," IEEE Signal Processing Letters, vol. 23, no. 5, pp. 610-614, 2016.

[32] J. Zheng, H. Pan, Q. Liu, and K. Ding, "Refined time-shift multiscale normalised dispersion entropy and its application to fault diagnosis of rolling bearing," Physica A: Statistical Mechanics and its Applications, vol. 545, Article ID 123641, 2019.

[33] H. Azami, M. Rostaghi, D. Abásolo, and J. Escudero, "Refined composite multiscale dispersion entropy and its application to biomedical signals," IEEE transactions on bio-medical engineering, vol. 99, p. 1, 2017.

[34] J. Zheng and H. Pan, "Use of generalized refined composite multiscale fractional dispersion entropy to diagnose the faults of rolling bearing," Nonlinear Dynamics, vol. 101, no. 2, pp. 1417-1440, 2020.

[35] H. Azami and J. Escudero, "Amplitude- and fluctuation-based dispersion entropy,” Entropy, vol. 20, no. 3, p. 210, 2018.

[36] Y. X. Li, S. B. Jiao, and X. Gao, "A novel signal feature extraction technology based on empirical wavelet transform and reverse dispersion entropy," Defence Technology, vol. 17, no. 5, pp. 1625-1635, 2021.

[37] L. Ma, M. Crawford, and J. Tian, "Local manifold learningbased k-nearest-neighbor for hyperspectral image classification," IEEE Transactions on Geoscience \& Remote Sensing, vol. 48, no. 11, pp. 4099-4109, 2010.

[38] X. Ran, X. Zhou, M. Lei, W. Tepsan, and W. Deng, “A novel $\mathrm{k}$-means clustering algorithm with a noise algorithm for capturing urban hotspots," Applied Sciences, vol. 11, no. 23, Article ID 11202, 2021. 